\title{
Schrödinger Operator on the Zigzag Half-Nanotube in Magnetic Field
}

\author{
A. Iantchenko ${ }^{1 *}$ and E. Korotyaev ${ }^{2}$ \\ ${ }^{1}$ Institute of Mathematics and Physics, Aberystwyth Univ., Penglais, Ceredigion, SY23 3BZ, UK ${ }^{\dagger}$ \\ ${ }^{2}$ School of Mathematics, Cardiff Univ., Senghennydd Road, Cardiff, CF24 4AG, UK
}

\begin{abstract}
We consider the zigzag half-nanotubes (tight-binding approximation) in a uniform magnetic field which is described by the magnetic Schrödinger operator with a periodic potential plus a finitely supported perturbation. We describe all eigenvalues and resonances of this operator, and theirs dependence on the magnetic field. The proof is reduced to the analysis of the periodic Jacobi operators on the half-line with finitely supported perturbations.
\end{abstract}

Key words: nanotubes, Jacobi operator, periodic, finite support perturbation, resonances AMS subject classification: 47B36, 81U15, 47N50, 81V99

\section{Introduction}

After their discovery [5], carbon nanotubes remain in both theoretical and applied research (see [19]). Structure of nanotubes are formed by rolling up a graphene sheet into a cylinder. Such nanomodels were introduced by Pauling [15] in 1936 to simulate aromatic molecules. They were described in more detail by Ruedenberg and Scherr [18] in 1953. Various physical properties of carbon nanotubes can be found in [19].

Single-wall nanotubes, one atomic layer in thickness in the radial direction, are a very important variety of carbon nanotube because they exhibit important electric properties that are not shared by the multi-walled carbon nanotube variants. Single-wall nanotubes are the most likely candidate

*Corresponding author. E-mail: ai@mah.se

† On leave from Malmö Högskola, Sweden 
for miniaturizing electronics beyond the micro electromechanical scale that is currently the basis of modern electronics.

We consider the Schrödinger operator $H^{b}=H_{0}^{b}+V+Q$ on the zigzag half-nanotube $\Gamma \subset \mathbb{R}^{3}$ (1D tight-binding model of zigzag single-wall half-nanotubes, see [19], [14]) in a uniform magnetic field $\mathscr{B}=|\mathscr{B}| \mathbf{e}_{0}, \mathbf{e}_{0}=(0,0,1) \in \mathbb{R}^{3}$. Here $H_{0}^{b}$ is the Hamiltonian of the nanotube in the magnetic field, $V$ is the periodic potential of the nanotube, $Q$ is the finitely supported perturbation.

There are numerous mathematical results about Schrödinger operators on carbon nanotubes (zigzag, armchair and chiral) (see for example [10], [11], [6], [12], [16]). All these papers consider the so called continuous models. But in the physical literature the most commonly used model is the tight-binding model. In the tight binding model for a solid-state lattice of atoms, it is assumed that the full Hamiltonian $\mathrm{H}$ of the system may be approximated by the Hamiltonian of an isolated atom centered at each lattice point. The mathematical models, e.g., the Schrödinger operator on the zigzag and armchair nanotubes and ribbons in a uniform magnetic field $\mathscr{B}$ and in an external periodic electric potential were considered in [8], [8], [16], see also [17]. For applications of our models see references in [1], [3], [19].

Our model nanotube $\Gamma$ is a graph (see Fig. 1. and 2) embedded in $\mathbb{R}^{3}$ oriented in the $z$-direction $\mathbf{e}_{0}$ with unit edge length. $\Gamma$ is a set of vertices (atoms) $\mathbf{r}_{\omega}$ connecting by bonds (edges) $\Gamma_{n, j, k}$ and

$$
\begin{aligned}
\Gamma=\cup_{\omega \in \mathcal{Z}} \mathbf{r}_{\omega}, \quad \mathbf{r}_{n, 0, k}=\varkappa_{n+2 k}+\frac{3 n}{2} \mathbf{e}_{0}, \quad \mathbf{r}_{n, 1, k}=\mathbf{r}_{n, 0, k}+\mathbf{e}_{0}, \\
\omega=(n, j, k) \in \mathcal{Z}=\mathbb{Z}_{+} \times\{0,1\} \times \mathbb{Z}_{N}, \quad \mathbb{Z}_{N}=\mathbb{Z} /(N \mathbb{Z}), \quad \varkappa_{k}=R\left(\cos \frac{\pi k}{N}, \sin \frac{\pi k}{N}, 0\right), \\
R=\frac{\sqrt{3}}{4 \sin \frac{\pi}{2 N}}, \quad \mathbb{Z}_{+}=\{j \in \mathbb{Z}, j \geqslant 0\} .
\end{aligned}
$$

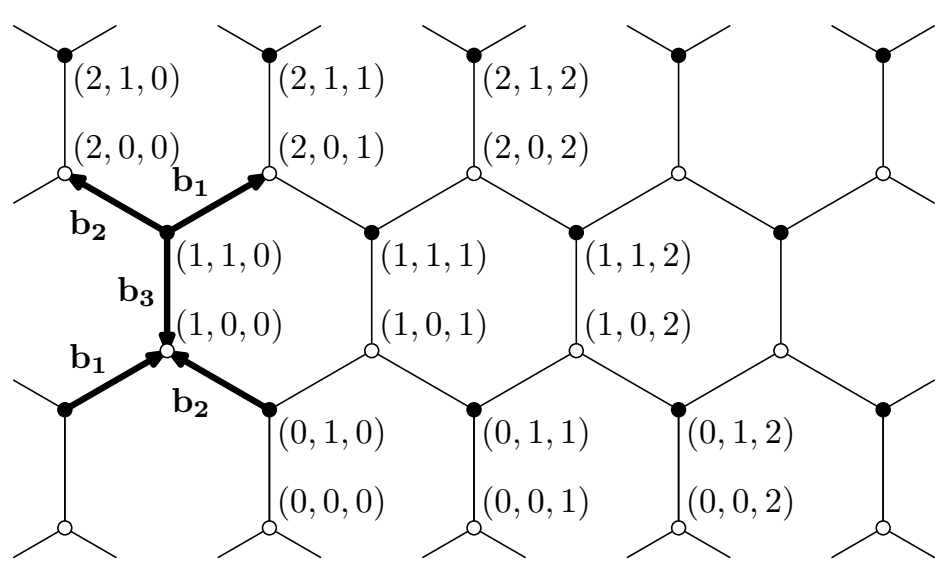

Fig 1. A piece of a nanotube.

Our carbon model nanotube is the honeycomb lattice of a graphene sheet rolled into a cylinder. This nanotube $\Gamma$ has $N$ hexagons around the cylinder embedded in $\mathbb{R}^{3}$. Here $n \in \mathbb{Z}$ labels the 
position in the axial direction of the tube, $j=0,1$ is a label for the two types of vertices (atoms) (see Fig. 1.), and $k \in \mathbb{Z}_{N}$ labels the position around the cylinder. The points $\mathbf{r}_{0,1, k}, k \in \mathbb{Z}_{N}$ are vertices of the regular $\mathrm{N}$-gon $\mathscr{P}_{0}$ and $\mathbf{r}_{1,0, k}$ are the vertices of the regular $\mathrm{N}$-gon $\mathscr{P}_{1}$. $\mathscr{P}_{1}$ arises from $\mathscr{P}_{0}$ by combination of the rotation around the axis of the cylinder $\mathcal{C}$ by the angle $\frac{\pi}{N}$ and of the translation by $\frac{1}{2} \mathbf{e}_{0}$. Repeating this procedure we obtain $\Gamma$.

Introduce the Hilbert space $\ell^{2}(\Gamma)$ of functions $f=\left(f_{\omega}\right)_{\omega \in \mathcal{Z}}$ on $\Gamma$ equipped with the norm $\|f\|_{\ell^{2}(\Gamma)}^{2}=\sum_{\omega \in \mathcal{Z}}\left|f_{\omega}\right|^{2}$. The tight-binding Hamiltonian $H^{b}$ on the half-nanotube $\Gamma$ has the form $H^{b}=H_{0}^{b}+\tilde{V}$ on $\ell^{2}(\Gamma)$, where $H_{0}^{b}$ is given by (see [11])

$$
\begin{aligned}
& \left(H_{0}^{b} f\right)_{n, 0, k}=e^{i b_{1}} f_{n-1,1, k}+e^{i b_{2}} f_{n-1,1, k+1}+e^{i b_{3}} f_{n, 1, k}, \quad f_{-1,1, k}=0, \\
& \left(H_{0}^{b} f\right)_{n, 1, k}=e^{i b_{1}} f_{n+1,0, k-1}+e^{i b_{2}} f_{n+1,0, k}+e^{-i b_{3}} f_{n, 0, k}, \quad f=\left(f_{\omega}\right)_{\omega \in \mathcal{Z}}, \\
& \omega=(n, j, k) \in \mathbb{Z}_{+} \times\{0,1\} \times \mathbb{Z}_{N}, \quad b_{3}=0, \quad b_{1}=-b_{2}=b=\frac{3|\mathscr{B}|}{16} \cot \frac{\pi}{2 N},
\end{aligned}
$$

and the operator $\tilde{V}=V+Q$ is given by

$$
(\tilde{V} f)_{\omega}=\tilde{V}_{\omega} f_{\omega}, \quad \text { where } \quad \tilde{V}_{n-1,1, k}=\tilde{v}_{2 n}, \quad \tilde{V}_{n, 0, k}=\tilde{v}_{2 n+1}, \quad \tilde{v}=\left(\tilde{v}_{n}\right)_{n \in \mathbb{N}} \in \ell^{\infty},
$$

where $\tilde{v}_{n}=v_{n}+q_{n}$ for $0 \leqslant n \leqslant p, q_{p} \neq 0$, and $\tilde{v}_{n}=v_{n}$ for $n>p$.

Such models can be realized using optical methods, by gating, or by an acoustic field (see [14]). For example, if an external potential is given by $A_{0} \cos \left(\xi_{0} z+\beta_{0}\right)$ for some constant $A_{0}, \xi_{0}, \beta_{0}$, then we obtain

$$
v_{2 n}=A \cos \left(2 \pi \xi\left(n-\frac{1}{3}\right)+\beta\right), \quad v_{2 n+1}=A \cos (2 \pi \xi n+\beta), \quad n \in \mathbb{N}=1,2, \ldots,
$$

for some constant $A, \xi, \beta$. If $\xi$ is rational, then the sequence $v_{n}$, is periodic for $n \in \mathbb{N}$. If $\xi$ is irrational, then the sequence $v_{n} n \in Z_{+}$is almost periodic.

We give the physical sense of the finitely supported potential $q=\left(q_{n}\right)_{n=0}^{\infty}, q_{p} \neq 0$ and $q_{n}=0$ for all $n>p$. There are two physical cases: a local defect in the nanotube and an effective potential. The effective potential is related to the boundary after cutting an infinite nanotube into two pieces. The effective potential is due to an imperfection in the structure of the half-nanotube near the cut and corresponds to perturbations $q$ with $p$ small. This motivates our detailed analysis of the properties of eigenvalues and resonances in the special case $p=1, p=2$, in Section 5 ..

In the present paper we suppose that the periodic background potential $v$ has period 2 and is given by $v_{2 n+1}=-v_{2 n}=v \in \mathbb{R}, n \in \mathbb{N}$.

We formulate the result proven in [9] in the form convenient for us.

Each operator $H^{b}, b \in \mathbb{R}$, is unitarily equivalent to the operator $\oplus_{1}^{N} J_{k}^{b}$, where $J_{k}^{b}$ is the Jacobi operator, acting on $\ell^{2}(\mathbb{N})$ and given by

$$
\begin{aligned}
& \left(J_{k}^{b} y\right)_{n}=a_{n-1} y_{n-1}+a_{n} y_{n+1}+\tilde{v}_{n} y_{n}, \quad(\text { for } n \geqslant 2), \quad\left(J_{k}^{b} y\right)_{1}=a_{1} y_{2}+\tilde{v}_{1} y_{1} \\
& a_{2 n} \equiv a_{k, 2 n}=2\left|c_{k}(b)\right|, \quad a_{2 n+1} \equiv a_{k, 2 n+1}=1, \quad c_{k}(b)=\cos \left(b+\frac{\pi k}{N}\right), n \in \mathbb{N}, \\
& \tilde{v}_{n}=v_{n}+q_{n}, \quad q_{j}=0 \text { for } j>p, q_{p} \neq 0,
\end{aligned}
$$




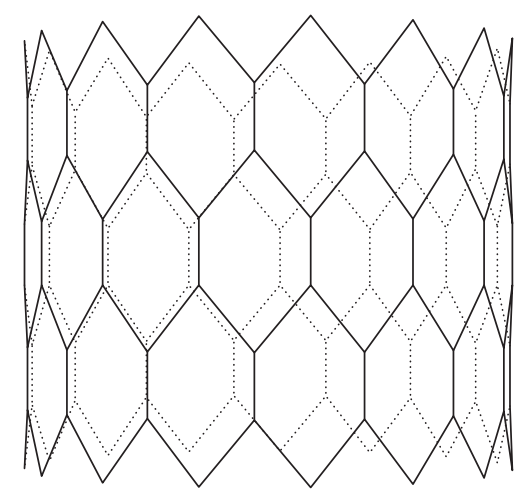

Fig 2. Nanotube in the magnetic field.

and $J_{k}^{b+\frac{\pi}{N}}=J_{k+1}^{b}, J_{k}^{-b}=J_{N-k}^{b}$ for all $(k, b) \in \mathbb{Z}_{N} \times \mathbb{R}$. Moreover, the operators $H^{ \pm b}$ and $H^{b+\frac{\pi}{N}}$ are unitarily equivalent for all $b \in \mathbb{R}$.

Remarks. 1) Note that the $n=1$ case in 1.4 can be thought of as forcing the Dirichlet condition $y(0)=0$. Thus, eigenfunctions must be non-vanishing at $n=1$ and eigenvalues must be simple.

2) The matrix of the operator $J_{k}^{b}$ is given by

$$
J_{k}^{b}=\left(\begin{array}{ccccc}
\tilde{v}_{1} & 1 & 0 & 0 & \cdots \\
1 & \tilde{v}_{2} & 2\left|c_{k}\right| & 0 & \cdots \\
0 & 2\left|c_{k}\right| & \tilde{v}_{3} & 1 & \cdots \\
0 & 0 & 1 & \tilde{v}_{4} & \cdots \\
0 & 0 & 0 & 2\left|c_{k}\right| & \cdots \\
\cdots & \cdots & \cdots & \cdots & \cdots
\end{array}\right)
$$

If $c_{k}=\cos \left(b+\frac{\pi k}{N}\right)=0$, then matrix (1.6) has the form

$$
\left.J_{k}^{b}\right|_{c_{k}=0}=\mathcal{J}=\left(\begin{array}{ccccc}
\tilde{v}_{1} & 1 & 0 & 0 & \ldots \\
1 & \tilde{v}_{2} & 0 & 0 & \ldots \\
0 & 0 & \tilde{v}_{3} & 1 & \ldots \\
0 & 0 & 1 & \tilde{v}_{4} & \ldots \\
0 & 0 & 0 & 0 & \ldots \\
\ldots & \ldots & \ldots & \ldots & \ldots
\end{array}\right)=\oplus_{n \in \mathbb{N}} \mathcal{J}_{n}, \mathcal{J}_{n}=\left(\begin{array}{cc}
\tilde{v}_{2 n-1} & 1 \\
1 & \tilde{v}_{2 n}
\end{array}\right)
$$

with the eigenvalues

$$
\left\{z_{n, \pm}=v_{n}^{+} \pm\left|v_{n}^{-2}+1\right|^{\frac{1}{2}}, \quad v_{n}^{ \pm}=\frac{\tilde{v}_{2 n-1} \pm \tilde{v}_{2 n}}{2}, \quad n \in \mathbb{N}\right\} .
$$

Moreover, if $q_{p} \neq 0$, then

if $p$ is even, then there are at most $p$ eigenvalues $z_{n, \pm}, n=1,2, \ldots, \frac{p}{2}$,

if $p$ is odd, then there are at most $p+1$ eigenvalues $z_{n, \pm}, n=1,2, \ldots, \frac{(p-1)}{2}$.

Note that for some special choice of perturbations $\left\{q_{1}, \ldots q_{p}\right\}$ we can have $z_{i}^{ \pm}=z_{j}^{ \pm}$for $i \neq j$. 
As perturbations have finite support, then there are always two flat bands (two eigenvalues with infinite multiplicities) given by $z_{n, \pm}= \pm\left|v^{2}+1\right|^{\frac{1}{2}}$, where $n \geqslant \frac{p}{2}+1$ if $p$ is even or $n \geqslant \frac{(p+1)}{2}+1$ if $p$ is odd. The flat bands are inherited from the pure periodic problem.

3) If $\left|c_{k}\right|=\frac{1}{2}$, then $J_{k}^{b}$ is the Schrödinger operator with $a_{n}=1$ for all $n \in \mathbb{N}$. In particular, if $b=0, \frac{N}{3} \in \mathbb{N}$, then $J_{\frac{N}{3}}^{0}$ is the Schrödinger operator.

4) Exner [2] obtained some duality between Schrödinger operators on graphs and certain Jacobi matrices, which depend on energy. In our case the Jacobi matrices do not depend on energy.

Unperturbed operator. We start with the unperturbed operator $H_{0}^{b}+V$, which is unitary equivalent to $\oplus_{1}^{N} J_{k}^{b, 0}$. The operator $J_{k}^{b, 0}$ is acting in $\ell^{2}(\mathbb{N})$ with Dirichlet boundary condition, see (1.4), where $\tilde{v}_{n}=v_{n}$ is the two-periodic potential verifying $v_{2 n+1}=-v_{2 n}=v \in \mathbb{R}$. It is known that, if $c_{k} \neq 0$, then the absolutely continuous spectrum of $J_{k}^{b, 0}$ is given by two bands and the bound states in $\gamma_{k}^{+}$(see Section 2.):

$$
\begin{array}{ll}
\sigma_{\mathrm{ac}}\left(J_{k}^{b, 0}\right)=\left[z_{k, 0}^{b,+}, z_{k, 0}^{b,-}\right] \backslash \gamma_{k, 1}^{b}, & \gamma_{k, 1}^{b}=\left(z_{k, 1}^{b,-}, z_{k, 1}^{b,+}\right), \\
z_{k, 0}^{b, \mp}= \pm \sqrt{v^{2}+\left(2\left|c_{k}\right|+1\right)^{2}}, & z_{k, 1}^{b, \pm}= \pm \sqrt{v^{2}+\left(2\left|c_{k}\right|-1\right)^{2}}, \quad k \in \mathbb{Z}_{N}, \\
\sigma\left(J_{k}^{b, 0}\right)=\sigma_{a c}\left(J_{k}^{b, 0}\right) \cup \sigma_{p p}\left(J_{k}^{b, 0}\right), & \sigma_{p p}\left(J_{k}^{b, 0}\right)= \begin{cases}\{v\} & \text { if } 1 / 2<\left|c_{k}\right| \leqslant 1, \\
\emptyset & \text { if } 0<\left|c_{k}\right| \leqslant 1 / 2,\end{cases}
\end{array}
$$

where $\gamma_{k, 1}^{b}$ is the middle gap in the spectrum of $J_{k}^{b, 0}$. We denote $\gamma_{0}=\left(-\infty, z_{k, 0}^{b,+}\right), \gamma_{2}=\left(z_{k, 0}^{b,-},+\infty\right)$ the infinite gaps.

We denote $\Lambda=\Lambda_{k}^{b}$ the two-sheeted Riemann surface for each $J_{k}^{b}$, obtained by joining the upper and low rims of two copies of the cut plane $\mathbb{C} \backslash \sigma_{\mathrm{ac}}\left(J_{k}^{b, 0}\right)$ in the usual (crosswise) way. For $j=0,1,2$, we denote the copies of $\gamma_{j}$ on $\Lambda^{+}$(respectively $\Lambda^{-}$) by $\gamma_{j}^{+}$(respectively $\gamma_{j}^{-}$), and put $\gamma_{j}^{\mathrm{c}}=\overline{\gamma_{j}^{+}} \cup \overline{\gamma_{j}^{-}}$. By abuse of notation we write also $\gamma_{j}$ for $\gamma_{j}^{+} \cup \gamma_{j}^{-}$and for its projection on $\mathbb{C}$.

If $0<\left|c_{k}\right|<1 / 2$, then $v \in \gamma_{1}^{-}$is an antibound state for $J_{k}^{b}$ and if $\left|c_{k}\right|=1 / 2$, then $v=z_{k, 1}^{b,+}$ or $v=z_{k, 1}^{b,-}$ is virtual state (see Definition 1 below and Proposition 6).

If $c_{k}=0$ for some $(k, b) \in \mathbb{Z}_{N} \times \mathbb{R}$, then (1.8) gives that the spectrum of $J_{k}^{b, 0}$ is pure point:

$$
\sigma\left(J_{k}^{b, 0}\right)=\sigma_{p p}\left(J_{k}^{b, 0}\right)=\left\{ \pm \sqrt{v^{2}+1}\right\}
$$

and each eigenvalue of $J_{k}^{b, 0}$ is a flat band, i.e. has infinite multiplicity.

In [9] it is shown that the spectral band $\left[z_{k, 0}^{b,+}, z_{k, 1}^{b,-}\right]$, (respectively $\left[z_{k, 1}^{b,+}, z_{k, 0}^{b,-}\right]$ ) shrinks to the flat band $-\sqrt{v^{2}+1}$ (respectively $\sqrt{v^{2}+1}$ ) as $c_{k} \rightarrow 0$ and the corresponding asymptotics are determined.

Let $b=\pi\left(\frac{1}{2}-\frac{1}{N}\right)$. Then $c_{1}=0$ and $c_{k}=\cos \pi\left(\frac{1}{2}-\frac{1}{N}+\frac{k}{N}\right)$ and the spectrum of $H^{b}$ is given by

$$
\begin{gathered}
\sigma\left(H^{b}\right)=\sigma_{a c}\left(H^{b}\right) \cup \sigma_{p p}\left(H^{b}\right), \quad \sigma_{p p}\left(H^{b}\right)=\left\{v, \pm \sqrt{1+v^{2}}\right\}, \\
\sigma_{a c}\left(H^{b}\right)=\left[z_{0}^{b,+}, z_{0}^{b,-}\right] \backslash \gamma\left(H^{b}\right), \gamma\left(H^{b}\right)=\left(z_{1}^{b,-}, z_{1}^{b,+}\right),
\end{gathered}
$$

where $\gamma\left(H^{b}\right)$ is the gap in the spectrum of $H^{b}$. If $b \neq \pi\left(\frac{1}{2}-\frac{1}{N}\right)$ and all $c_{k} \neq 0, k=1, \ldots, N$, then we obtain $\left\{ \pm \sqrt{1+v^{2}}\right\} \notin \sigma_{p p}\left(H^{b}\right)$. 
Note that if $c_{k}=0$ for some $k \in \mathbb{Z}_{N}$ then $\sigma_{p p}\left(H^{b}\right)=\left\{ \pm \sqrt{1+v^{2}}\right\} \subset \gamma\left(H^{b}\right)$. From [9] we know that $\sigma\left(H^{b+\frac{\pi}{N}}\right)=\sigma\left(H^{b}\right)$ for all $b \in \mathbb{R}$. Then we need to consider only the case $b \in\left[0, \frac{\pi}{N}\right)$ and in this case we get

$$
z_{0}^{b,+}=\left\{\begin{array}{lll}
z_{0,0}^{b,+} & \text { if } & b \leqslant \frac{\pi}{2 N} \\
z_{N-1,0}^{b,+} & \text { if } & b>\frac{\pi}{2 N}
\end{array},\right.
$$

Moreover, in particular case $\mathscr{B}=0, \frac{N}{3} \in \mathbb{N}, b=0$, we obtain $\gamma\left(H^{0}\right)=(-|v|,|v|)$.

Finitely supported perturbations. We consider the main operator $H^{b}=H_{0}^{b}+V+Q$. Recall that $H^{b}$ is unitary equivalent to $\oplus_{1}^{N} J_{k}^{b}$, where $J_{k}^{b}=J_{k}^{b, 0}+q$ is given by (1.4) with $q_{n}=0$ for $n>p$ and the sequence $y=\left(y_{n}\right)_{n=0}^{\infty}$ satisfies the Dirichlet boundary condition $y_{0}=0$.

The perturbation $q$ does not change the absolutely continuous spectrum: $\sigma_{\mathrm{ac}}\left(J_{k}^{b}\right)=\sigma_{\mathrm{ac}}\left(J_{k}^{b, 0}\right)=$ $\left[\lambda_{0}^{+}, \lambda_{1}^{-}\right] \cup\left[\lambda_{1}^{+}, \lambda_{0}^{-}\right]$, where we used the simplified notations $\lambda_{0}^{ \pm} \equiv z_{k, 0}^{b, \pm}$ and $\lambda_{1}^{ \pm} \equiv z_{k, 1}^{b, \pm}$.

In our paper we study the global properties of eigenvalues, virtual states and resonances of $J=J_{k}^{b}$. Let $R(\lambda)=(J-\lambda)^{-1}$ denote the resolvent of $J$ and let $\langle\cdot, \cdot\rangle$ denote the scalar product in $\ell^{2}(\mathbb{N})$. Then for any $f, g \in \ell^{2}(\mathbb{N})$ the function $\langle R f, g\rangle$ is defined on $\Lambda_{+}$outside the poles at the bound states $\lambda_{0} \in \gamma_{j}^{+}, j=0,1,2$. Recall that the bound states are simple. Moreover, if $f, g \in \ell_{\text {comp }}^{2}(\mathbb{N})$, where $\ell_{\text {comp }}^{2}(\mathbb{N})$ denotes the $\ell^{2}$ functions on $\mathbb{N}$ with finite support, then the function $\langle R f, g\rangle$ has an analytic extension from $\Lambda_{+}$into the Riemann surface $\Lambda$.

Definition 1. Let $c_{k}(b) \neq 0$ for some $b \in \mathbb{R}$.

1) A number $\lambda_{0} \in \Lambda_{-}$is a resonance, if the function $\langle R f, g\rangle$ has a pole at $\lambda_{0}$ for some $f, g \in$ $\ell_{\text {comp }}^{2}(\mathbb{N})$. The multiplicity of the resonance is the multiplicity of the pole. If $\operatorname{Re} \lambda_{0}=0$, we call $\lambda_{0}$ antibound state.

2) A real number $\lambda_{0}=\lambda_{0}^{ \pm}$or $\lambda_{0}=\lambda_{1}^{ \pm}$is a virtual state if $\langle R f, g\rangle$ has a singularity at $\lambda_{0}$ for some $f, g \in \ell_{\text {comp }}^{2}(\mathbb{N})$.

3) The state $\lambda \in \Lambda$ is a bound state or a resonance or a virtual state of $J$.

We denote the set of all states of $J$ by $\mathfrak{S}(J)$.

In Section 3., 8, we give an equivalent characterization of the states.

In the unperturbed case $J^{0}=J_{k}^{b, 0}$ we show in Proposition 6 that if $0<c_{k} \leqslant 1$, then $\mathfrak{S}\left(J^{0}\right)$ consists of one state: a bound state $v \in \gamma_{1}^{+}$, a antibound state $v \in \gamma_{1}^{-}$or a virtual state $v=\lambda_{1}^{ \pm}$. Note that any such state is projected on the Dirichlet eigenvalue $v \in \mathbb{C}, \varphi_{2}(v)=0$.

Let $\vartheta_{n}, \varphi_{n}$ be the fundamental solutions of the equation $a_{n-1} y_{n-1}+a_{n} y_{n+1}+v_{n} y_{n}=\lambda y_{n}$, satisfying $\vartheta_{0}=\varphi_{1}=1, \vartheta_{1}=\varphi_{0}=0$. Let $f_{n}^{ \pm}$be the Jost solution, $f_{n}^{ \pm}=\tilde{\vartheta}_{n}+m_{ \pm} \tilde{\varphi}_{n}$, where $\tilde{\vartheta}_{n}, \tilde{\varphi}_{n}$ denote the solutions to (1.4) satisfying $\tilde{\vartheta}_{n}=\vartheta_{n}, \tilde{\varphi}_{n}=\varphi_{n}$, for $n>p$. Here $m_{ \pm}$are the Titchmarch-Weyl functions. The functions $\varphi, \vartheta$ are polynomials, the Jost solutions $f^{ \pm}$and functions $m_{ \pm}$are meromorphic functions on $\Lambda$. Note that $f^{-}(\lambda)=\overline{f^{+}(\bar{\lambda})}, \lambda \in \Lambda$, and $f^{ \pm}(\lambda) \in \ell^{2}(\mathbb{N})$ for any $\lambda \in \Lambda_{ \pm}$. We call $f_{0}^{ \pm}$the Jost functions.

We pass to the formulation of main results of the present paper. Recall that all bound and virtual states of $J \equiv J_{k}^{b}$ are simple (see Lemma 11). In the next theorem we give the characterization of the states of $J_{k}^{b}$. 
Theorem 2. Let $c_{k}(b) \neq 0$.

i) The point $\lambda=v \in \gamma_{1}^{+}$or $\lambda=v \in \gamma_{1}^{-}$is a state of $J=J_{k}^{b}$ iff the projection of $\lambda$ on $\mathbb{C}$ is a zero of $\tilde{\varphi}_{0}$. The value $\lambda \in \Lambda$ whose projection on the complex plane does not coincide with $v$ is a state of $J$ iff $\lambda \in \Lambda$ is a zero of the Jost function $f_{0}^{+}$:

$$
\left.\mathfrak{S}(J) \backslash\{v\}=\left\{\lambda \in \Lambda: f_{0}^{+}(\lambda)=0\right)\right\} \subset\left(\cup_{j=0,1,2} \overline{\gamma_{j}^{ \pm}}\right) \cup \Lambda_{-} .
$$

ii) The state $\lambda=\lambda_{0,1}^{ \pm}$is a virtual state of $J$ iff one of the following two conditions is satisfied: 1) $\lambda \neq v$ and $f_{0}^{+}(\lambda)=0$; 2) $\lambda=v$ and $\tilde{\varphi}_{0}(\lambda)=0$.

iii) If $\lambda=v \in \gamma_{1}^{-}$is an antibound state for $J$ then it is necessarily simple.

The distribution of the states is summarized in the following theorem.

Theorem 3. Let $c_{k}(b) \neq 0, q_{p} \neq 0$. Then the Jacobi operator $J \equiv J_{k}^{b}$ has $2 p$ states counted with multiplicities. Moreover, the following facts hold true.

1) The total number of bound states and virtual states is $\geqslant 2$.

2) In the closure of the middle gap $\gamma_{1}^{\mathrm{c}}=\overline{\gamma_{1}^{+}} \cup \overline{\gamma_{1}^{-}}$there is always an odd number of states with at least one bound or virtual state.

3) Let $\lambda_{1}<\lambda_{2}$ be any two bound states of $J, \lambda_{1,2} \in \gamma_{k}^{+}$, for some $k=0,1,2$, such that there are no other eigenvalues on the interval $\Omega^{+}=\left(\lambda_{1}, \lambda_{2}\right) \subset \gamma_{k}^{+}$. Then there exists an odd number $\geqslant 1$ of antibound states on $\Omega^{-}$, where $\Omega^{-} \subset \gamma_{k}^{-} \subset \Lambda_{-}$is the same interval but on the second sheet.

Remarks. 1) If all $c_{k} \neq 0$ and $q_{p} \neq 0$, then the operator $\oplus_{1}^{N} J_{k}^{b}$ has in total $N 2 p$ states.

2) If $p$ is even and $q_{1}=q_{3}=\ldots=q_{p-1}=0$, then $\{v\}$ is always a bound state or antibound state (see Lemma 13).

In Theorem 4 we consider the limit of the states of each $J_{k}^{b}$ as $c_{k} \rightarrow 0$. Recall that operator $\left.J_{k}^{b}\right|_{c_{k}=0}$ has two flat bands and a finite number of simple eigenvalues.

Theorem 4. Let $z_{n, \pm}, n \in \mathbb{N}$, be the eigenvalues of the matrix $\left.J_{k}^{b}\right|_{c_{k}=0}$ given in (1.7). Let $c_{k} \rightarrow 0+$. 1) If $p$ is even, then

a) the set of bound states of $J_{k}^{b}$ converges to the set $\left\{z_{n, \pm}, n=1, \ldots, \frac{p}{2}\right\} \subset \mathbb{R}$,

$b)$ the set of all resonances of $J_{k}^{b}$ converges to the set of numbers $\left\{z_{n, \pm}, n=1, \ldots, \frac{(p-2)}{2}\right\} \cup\left\{\mu_{p-1}^{0}, \mu_{p}^{0}\right\}$, where only the numbers

$$
\mu_{p-1, p}^{0}=v+\frac{q_{p-1}}{2} \pm \sqrt{\frac{q_{p-1}^{2}}{4}-\frac{q_{p-1}}{q_{p}}},
$$

can be complex.

2) If $p$ is odd, then

a) the set of bound states of $J_{k}^{b}$ converges to the set $\left\{z_{n, \pm}, n=1, \ldots, \frac{(p+1)}{2}\right\} \subset \mathbb{R}$;

b) the set of resonances of $J_{k}^{b}$ converge to the set of real numbers $\left\{z_{n, \pm}, n=1, \ldots, \frac{(p-1)}{2}\right\}$. 
In Theorem 5 we consider the asymptotics of the states of the half-nanotube Hamiltonian $H^{b}$ (unitary equivalent to $\oplus_{1}^{N} J_{k}^{b}$ ) for large perturbation.

Theorem 5. Suppose $q_{j}=q_{j}^{0} t, j=1, \ldots, p$, where all $q_{j}^{0} \neq 0$ are fixed and $t>1$. If $\lambda(t) \in \Lambda$ is a state of $H^{b}$, then either $|\lambda(t)| \rightarrow \infty$ or $\lambda(t) \rightarrow(-1)^{p}$ v as $t \rightarrow \infty$.

If $v \rightarrow \infty$, then (1.9) implies that the absolutely continuous spectrum degenerates into two points $\{v\},\{-v\}$.

Suppose $p=2$ and $q_{1}=0$ and $q_{2}$ is small enough, then the Hamiltonian $H^{b}$ has precisely $2 N$ non-real complex conjugated resonances. More results about the cases $p=1$ and $p=2$ are given in Section 5..

The plan of the paper is as follows. In Section 2. we collect some well known facts about the two-periodic Jacobi operators and its perturbations in the form convenient for us.

In Section 3. we describe the properties of the perturbed operator.

In Section 4. we consider the properties of the polynomial $F=\varphi_{2} f_{0}^{+} f_{0}^{-}$which plays the crucial role in the proof of the main results, similar to the case [7]. Theorem 3 follows from Lemma 10 and Theorem 2 follows from Lemmata 11 and 12. Theorems 4 and 5 follows from Lemmata 7 and 9. In Section 5. we consider the cases $p=1$ and $p=2$.

\section{Periodic Jacobi operator.}

In this section we recall some well known facts about the infinite Jacobi matrix $\mathbb{J}^{0}$

$$
\left(\begin{array}{ccccccc}
\ldots & \ldots & \cdots & \ldots & \ldots & \ldots & \ldots \\
\ldots & a & v & 1 & 0 & 0 & \ldots \\
\ldots & 0 & 1 & -v & a & 0 & \ldots \\
\ldots & 0 & 0 & a & v & 1 & \ldots \\
\ldots & 0 & 0 & 0 & 1 & -v & \ldots \\
\ldots & 0 & 0 & 0 & 0 & a & \ldots \\
\ldots & \ldots & \ldots & \ldots & \ldots & \ldots & \ldots
\end{array}\right), \quad 0<a \leqslant 2
$$

and the associated equation for $\mathbb{J}^{0}$

$$
a_{n-1} y_{n-1}+a_{n} y_{n+1}+v_{n} y_{n}=\lambda y_{n}, \quad a_{2 n+1}=1, a_{2 n}=a, v_{2 n+1}=v, v_{2 n}=-v,
$$

$(\lambda, n) \in \mathbb{C} \times \mathbb{Z}$. Introduce fundamental solutions $\varphi=\left(\varphi_{n}(z)\right)_{n \in \mathbb{Z}}$ and $\vartheta=\left(\vartheta_{n}(z)\right)_{n \in \mathbb{Z}}$ for equation (2.2), under the condition $\vartheta_{0}=\varphi_{1}=1$ and $\vartheta_{1}=\varphi_{0}=0$. We obtain

$$
\begin{aligned}
& \varphi_{0}=0, \quad \varphi_{1}=1, \quad \varphi_{2}=\lambda-v, \quad \varphi_{3}=\frac{\lambda^{2}-v^{2}-1}{a}, \\
& \vartheta_{0}=1, \quad \vartheta_{1}=0, \quad \vartheta_{2}=-a, \quad \vartheta_{3}=-\lambda-v \quad \ldots .
\end{aligned}
$$

The monodromy matrix $M_{2}$ satisfies

$$
M_{2}(\lambda)=\left(\begin{array}{ll}
\vartheta_{2} & \varphi_{2} \\
\vartheta_{3} & \varphi_{3}
\end{array}\right)=\left(\begin{array}{cc}
-a & \lambda-v \\
-\lambda-v & \frac{\lambda^{2}-v^{2}-1}{a}
\end{array}\right) .
$$


The Lyapunov function is defined in the standard way:

$$
\Delta=\frac{\operatorname{Tr} M_{2}}{2}=\frac{\lambda^{2}-v^{2}-a^{2}-1}{2 a}=\cos 2 \varkappa,
$$

where $\varkappa$ is the Bloch quasimomentum.

The periodic eigenvalues $\lambda_{0}^{ \pm}$satisfy the equation $\Delta(\lambda)=1$ and the anti-periodic eigenvalues $\lambda_{1}^{ \pm}$satisfy the equation $\Delta(\lambda)=-1$ and they are given by

$$
\lambda_{0}^{\mp}= \pm \sqrt{v^{2}+(a+1)^{2}}, \quad \lambda_{1}^{ \pm}= \pm \sqrt{v^{2}+(a-1)^{2}} .
$$

The absolutely continuous spectrum of $\mathbb{J}^{0}$ has the form

$$
\sigma_{\mathrm{ac}}\left(\mathbb{J}^{0}\right)=\left[\lambda_{0}^{+}, \lambda_{1}^{-}\right] \cup\left[\lambda_{1}^{+}, \lambda_{0}^{-}\right]=\left[\lambda_{0}^{+}, \lambda_{0}^{-}\right] \backslash \gamma_{1}, \quad \gamma_{1}=\left(\lambda_{1}^{-}, \lambda_{1}^{+}\right)
$$

where $\gamma_{1}$ is a gap. Note that $\gamma_{1}=\left(\lambda_{1}^{-}, \lambda_{1}^{+}\right) \neq \emptyset$, if $|v|+|a-1|>0$. We denote also $\gamma_{0}=\left(-\infty, \lambda_{0}^{+}\right)$and $\gamma_{2}=\left(\lambda_{0}^{-},+\infty\right)$.

We recall from the Introduction that the two-sheeted Riemann surface $\Lambda$ is obtained by joining the upper and low rims of two copies $\Lambda^{ \pm}$of the cut plane $\mathbb{C} \backslash \sigma_{\mathrm{ac}}\left(\mathbb{J}^{0}\right)$ in the usual (crosswise) way. For $j=0,1,2, \gamma_{j}^{+}$(respectively $\gamma_{j}^{-}$) denote the copies of $\gamma_{j}$ on $\Lambda^{+}$(respectively $\Lambda^{-}$), and $\gamma_{j}^{\mathrm{c}}=\overline{\gamma_{j}^{+}} \cup \overline{\gamma_{j}^{-}}$. By abuse of notation we write also $\gamma_{j}$ for $\gamma_{j}^{+} \cup \gamma_{j}^{-}$and for its projection on $\mathbb{C}$.

The eigenvalues of $M_{2}$ are given by $\xi_{ \pm}^{2}=\Delta \pm \sqrt{\Delta^{2}-1}$. On $\gamma_{0}^{+}$, we choose

$$
\text { for } \lambda \in \gamma_{0}^{+}=\left(-\infty, \lambda_{0}^{+}\right) \subset \Lambda^{+}, \xi_{+}^{2}=\Delta-\sqrt{\Delta^{2}-1}, \xi_{-}^{2}=\Delta+\sqrt{\Delta^{2}-1} .
$$

For others $\lambda \in \Lambda$, the functions $\xi_{ \pm}(\lambda)$ are defined by an analytic continuation.

If $\lambda= \pm v \in \gamma_{1}^{+}$(these numbers will play a special role later) then $\Delta( \pm v)=\frac{-a^{2}-1}{2 a}$ and

$$
\xi_{+}^{2}( \pm v)=\frac{-a^{2}-1}{2 a}+\left|\frac{a^{2}-1}{2 a}\right|= \begin{cases}-a & \text { if } 0<a<1 \\ -1 / a & \text { if } a>1\end{cases}
$$

and opposite for $\xi_{-}^{2}( \pm v)$.

Then for $\lambda \in \gamma_{0} \cup \gamma_{1} \cup \gamma_{2}$ we have $\left|\xi_{+}^{2}\right|<1$ and $\left|\xi_{-}^{2}\right|>1$. The eigenvectors of $M_{2}$ are chosen in the form $\left(1, m_{ \pm}\right)$and then the Titchmarsh-Weyl functions are

$$
m_{ \pm}(\lambda)=\frac{\xi_{ \pm}^{2}-\vartheta_{2}}{\varphi_{2}}=\frac{\xi_{ \pm}^{2}+a}{\lambda-v}
$$

For $\lambda \in \gamma_{1}^{+}$we have also

$$
\begin{aligned}
m_{ \pm} & =\frac{\phi \pm \sqrt{\Delta^{2}-1}}{\varphi_{2}}=\frac{\Delta+a \pm \sqrt{\Delta^{2}(\lambda)-1}}{\lambda-v}=\frac{\phi \pm i \sin 2 \varkappa}{\lambda-v} \\
\phi & =\frac{\varphi_{3}-\vartheta_{2}}{2}=\frac{\lambda^{2}-v^{2}+a^{2}-1}{2 a}=\Delta+a .
\end{aligned}
$$


On each $\gamma_{k}^{+}, k=0,1,2$, the quasimomentum $\varkappa(\lambda)$ has constant positive imaginary part and we put $\varkappa=i h, h=h_{k}>0$. Then $\Delta=\cosh (2 h)$ and

$$
i \sin 2 \varkappa=-(-1)^{k} \sqrt{\Delta^{2}(\lambda)-1}=-(-1)^{k} \sinh 2 h .
$$

Now the Floquet solutions $\psi_{n}^{ \pm}=\vartheta_{n}+m_{ \pm} \varphi_{n}$ are

$$
\psi_{0}^{ \pm}=1, \quad \psi_{1}^{ \pm}=m_{ \pm}, \quad \psi_{2}^{ \pm}=e^{ \pm 2 i \varkappa}=\xi_{ \pm}^{2}, \quad \psi_{2 n}^{ \pm}=\xi_{ \pm}^{2 n}, \quad \psi_{2 n+1}^{ \pm}=\xi_{ \pm}^{2 n} m_{ \pm},
$$

where $\xi_{ \pm}^{2}=e^{ \pm 2 i \varkappa}$ are the Floquet multipliers. Recall that $\psi_{n}^{ \pm} \in \ell^{2}(\mathbb{N})$ for any $\lambda \in \Lambda^{ \pm}$.

Note the following simple identities which will be used in the paper:

$$
\phi^{2}+1-\Delta^{2}=1-\varphi_{3} \vartheta_{2}=-\vartheta_{3} \varphi_{2} .
$$

Let $\left\{\phi_{n}, \psi_{n}\right\}=a_{n}\left(\phi_{n} \psi_{n+1}-\phi_{n+1} \psi_{n}\right\}$ denote the Wronskian.

In the next theorem we describe the states of the restriction of $\mathbb{J}^{0}$ to $\mathbb{N}$ defined in (1.4) with $\tilde{v}_{n}=v_{n}$.

Proposition 6 (Unperturbed case). The half-periodic Jacobi operator $J^{0}$ given by equation (1.4) with $\tilde{v}_{n}=v_{n}$, has absolutely continuous spectrum (2.7): $\sigma_{\mathrm{ac}}\left(J^{0}\right)=\left[\lambda_{0}^{+}, \lambda_{1}^{-}\right] \cup\left[\lambda_{1}^{+}, \lambda_{0}^{-}\right]$and a state at $\lambda=v \in \overline{\gamma_{1}^{+}} \cup \overline{\gamma_{1}^{-}}$, whose projection $v \in \mathbb{C}$ satisfies $\varphi_{2}(v)=0$. There are three possibilities: if $a>1$ then $J^{0}$ has simple bound state at $\lambda=v \in \gamma_{1}^{+}$;

if $0<a<1$ then $J^{0}$ has simple antibound state at $\lambda=v \in \gamma_{1}^{-}$;

if $a=1$ then $\lambda=v$ is a simple virtual state, $v=\lambda_{1}^{+}$or $v=\lambda_{1}^{-}$if $v>0$ respectively $v<0$.

Proof: The kernel of the resolvent of $J^{0}$ is given by

$$
R_{0}(n, m)=-\frac{\varphi_{n} \psi_{m}^{+}}{\left\{\varphi, \psi^{+}\right\}}, n<m
$$

where $\left\{\varphi, \psi^{+}\right\}=-a$. According to Lemma 8 (see Section 3.), the bound states (resonances) are the poles of $\mathscr{R}_{0}(n)=\psi_{n}^{+}(\lambda)=\vartheta_{n}(\lambda)+m_{+}(\lambda) \varphi_{n}(\lambda)$ on $\Lambda_{+}$(respectively on $\Lambda_{-}$). Hence, the only state is the pole of $m_{+}$on $\Lambda_{ \pm}$, whose projection on $\mathbb{C}$ is the zero of $\varphi_{2}(\lambda)$, i.e. $\lambda=v \in \gamma_{1}$.

We have

$$
m_{+}=\frac{\xi_{+}^{2}+a}{\lambda-v}, a=2\left|c_{k}\right|, c_{k}=\cos \left(b+\frac{\pi k}{N}\right) .
$$

If $0<a<1$, then by (2.9)) $\lambda=v \in \gamma_{1}^{+}$is a simple zero for the numerator while at $\lambda=v \in \gamma_{1}^{-}$ the numerator is non-zero. Thus $\lambda=v$ is an antibound state. Similar we get that if $1<a<2$, then $\lambda=v$ is a bound state.

If $a=1$ then $\Delta=\left(\lambda^{2}-v^{2}-2\right) / 2$ and

$$
\Delta^{2}-1=-(\lambda-v)(\lambda+v)+\frac{(\lambda-v)^{2}(\lambda+v)^{2}}{4} .
$$

Suppose $v>0$, then $v=\lambda_{1}^{+}$. Let $\lambda-v=-\epsilon, \epsilon>0$, and let $\epsilon \rightarrow 0$. Then

$$
\Delta=-1-v \epsilon+\mathcal{O}\left(\epsilon^{2}\right), \sqrt{\Delta^{2}-1}=\sqrt{\epsilon} \sqrt{2 v}+\mathcal{O}(\epsilon),
$$


and

$$
m_{+}(v-\epsilon)=\frac{\Delta+a+\sqrt{\Delta^{2}-1}}{\lambda-v}=\frac{\sqrt{\epsilon} \sqrt{2 v}+\mathcal{O}(\epsilon)}{\epsilon}=\frac{\sqrt{2 v}}{\sqrt{\epsilon}}+\mathcal{O}(1) .
$$

Thus if $a=1$, the function $\left(\mathscr{R}_{n}(\lambda)\right)^{2}$ has a pole at $\lambda=v$ and $\lambda=v$ is a virtual state.

\section{Jost functions}

We introduce the Jost solutions as solutions $f_{n}^{ \pm}$, of the equation

$$
a_{n-1} y_{n-1}+a_{n} y_{n+1}+\tilde{v}_{n} y_{n}=\lambda y_{n}, \quad n \in \mathbb{N}, \quad \lambda \in \Lambda,
$$

satisfying

$$
f_{n}^{ \pm}=\psi_{n}^{ \pm}, \text {for } n>p
$$

where $\psi_{n}^{ \pm}$are the Floquet solutions (2.14) for the unperturbed problem, and $\tilde{v}_{j}=v_{j}+q_{j}$ with $q_{n}=0$ for $n>p$. We recall that, as in (2.2), we have $v_{2 n+1}=-v_{2 n}=v \in \mathbb{R}, a_{2 n+1}=1, a_{2 n}=$ $a=2\left|c_{k}\right| \neq 0, c_{k}=\cos \left(b+\frac{\pi k}{N}\right)$. We have $\overline{f_{n}^{ \pm}}(\bar{\lambda})=f_{n}^{\mp}(\lambda), \lambda \in \Lambda$.

The equation (3.1) has unique solutions $\tilde{\vartheta}_{n}, \tilde{\varphi}_{n}$ such that

$$
\tilde{\vartheta}_{n}(\lambda)=\vartheta_{n}(\lambda), \quad \tilde{\varphi}_{n}(\lambda)=\varphi_{n}(\lambda) \text { for } n>p, \lambda \in \mathbb{C} .
$$

The functions $\tilde{\vartheta}_{n}(\cdot), \tilde{\varphi}_{n}(\cdot)$ are polynomials. The functions $f_{n}^{ \pm}$have the form

$$
f_{n}^{ \pm}=\tilde{\vartheta}_{n}+m_{ \pm} \tilde{\varphi}_{n}, m_{ \pm}=\frac{\phi \pm i \sin 2 \varkappa}{\varphi_{2}}=\frac{\Delta+a \pm \sqrt{\Delta^{2}(\lambda)-1}}{\lambda-v} .
$$

Here $\phi$ is defined in (2.12), $\varphi_{2}=\lambda-v$ and $\Delta$ is the Lyapunov function. The functions $f_{0}^{ \pm}$are called Jost functions. The Jost functions are analytic at all $\lambda \in \Lambda$ whose projection on the complex plane $\mathbb{C}$ is different from $v$, and has branch points $\lambda_{0,1}^{ \pm}$.

The asymptotics of the Jost functions are given in the following Lemma.

Lemma 7. Let $p, n \in \mathbb{N}$ and $p \geqslant n$. Suppose $q_{p} \neq 0$.

1) If $p$ is even $\left(a_{p}=a, v_{p}=-v\right)$, then

for $\lambda \in \gamma_{0,2}^{+}$in the limit $|\lambda| \rightarrow \infty$, we have

$$
f_{0}^{+}=1-\lambda^{-1} \sum_{k=1}^{p} q_{k}+\mathcal{O}\left(\lambda^{-2}\right), \quad f_{0}^{-}=\frac{\lambda^{2 p-1}}{a^{p}}\left[-q_{p}+\mathcal{O}\left(\lambda^{-1}\right)\right]
$$

for $\lambda \in \gamma_{1}^{+}$as $a \rightarrow 0+$, we have

$$
\begin{aligned}
& f_{0}^{+}=(2 \delta)^{-p / 2} \prod_{k=1}^{p-1}\left[\left(\lambda-\tilde{v}_{k}\right)\left(\lambda-\tilde{v}_{k+1}\right)-1\right]+\delta^{-p / 2} \mathcal{O}\left(a^{2}\right) \\
& f_{0}^{-}=\frac{(2 \delta)^{p / 2}}{a^{p}}\left[\left(\lambda-\tilde{v}_{p-1}\right)\left\{\left(\lambda-\tilde{v}_{p}\right)-\frac{2 \delta}{\lambda-v}\right\}-1\right] \prod_{k=1}^{p-3}\left[\left(\lambda-\tilde{v}_{k}\right)\left(\lambda-\tilde{v}_{k+1}\right)-1\right] \\
& +\delta^{p / 2} \mathcal{O}\left(a^{2}\right),
\end{aligned}
$$


where $\delta=\left(\lambda^{2}-v^{2}-1\right) / 2$;

if $q_{k}=t q_{k}^{0}$ with all $q_{k}^{0} \neq 0$ and $t \rightarrow \infty$, then we have $f_{0}^{+}=t^{p} \frac{\xi_{+}^{p}}{a^{p / 2}} \prod_{k=1}^{p} q_{k}^{0}+\mathcal{O}\left(t^{p-1}\right)$.

2) If $p$ is odd $\left(a_{p}=1, v_{p}=v\right)$, then

for $\lambda \in \gamma_{0,2}^{+}$in the limit $|\lambda| \rightarrow \infty$, we have

$$
f_{0}^{+}=1-\lambda^{-1} \sum_{k=1}^{p} q_{k}+\mathcal{O}\left(\lambda^{-2}\right), \quad f_{0}^{-}=\frac{\lambda^{2 p-1}}{a^{p+1}}\left[-q_{p}+\mathcal{O}\left(\lambda^{-1}\right)\right]
$$

for $\lambda \in \gamma_{1}^{+}$, as $a \rightarrow 0+$, we have

$$
\begin{aligned}
& f_{0}^{+}=(2 \delta)^{-(p+1) / 2}\left[\left(\lambda-\tilde{v}_{p}\right)(\lambda+v)-1\right] \prod_{k=1}^{p-2}\left[\left(\lambda-\tilde{v}_{k}\right)\left(\lambda-\tilde{v}_{k+1}\right)-1\right]+\delta^{-(p+1) / 2} \mathcal{O}\left(a^{2}\right), \\
& f_{0}^{-}=\frac{(2 \delta)^{(p+1) / 2}}{a^{p+1}} \cdot \frac{-q_{p}}{\lambda-v} \prod_{k=1}^{p-2}\left[\left(\lambda-\tilde{v}_{k}\right)\left(\lambda-\tilde{v}_{k+1}\right)-1\right]+\delta^{(p+1) / 2} \mathcal{O}\left(a^{2}\right),
\end{aligned}
$$

if $q_{k}=t q_{k}^{0}$ with $q_{k}^{0} \neq 0$ and $t \rightarrow \infty$, then $f_{0}^{+}=t^{p} \frac{\xi_{+}^{p}}{a^{(p+1) / 2}} \frac{1+a \xi_{-}^{2}}{\lambda-v} \prod_{k=1}^{p} q_{k}^{0}+\mathcal{O}\left(t^{p-1}\right)$.

The proof is technical and uses the standard arguments. The asymptotics of $f_{0}^{+}$on $\gamma_{0,2}^{+}$as $\lambda \rightarrow \infty$ are well known (see for example Teschl [20]).

It is well known that the spectrum of $J=J_{k}^{b}$, introduced in (1.4), consists of absolutely continuous part $\sigma_{\mathrm{ac}}(J)=\sigma_{\mathrm{ac}}\left(J^{0}\right)$ and a finite number of simple bound states in each gap $\gamma_{k}^{+}, k=0,1,2$. The states of $J$ correspond to the poles of a meromorphic function: resolvent or its square.

The kernel of the resolvent of $J$ is

$$
R(n, m)=\left\langle e_{n},(J-\lambda)^{-1} e_{m}\right\rangle=-\frac{\Phi_{n} f_{m}^{+}}{\left\{\Phi, f^{+}\right\}}, n<m,
$$

where $e_{n}=\left(\delta_{n, j}\right)_{j \in \mathbb{N}}, J \Phi_{n}=\lambda \Phi_{n}, \Phi_{0}=0, \Phi_{1}=1$, and the Wronskian $\left\{\Phi, f^{+}\right\}=-a_{0} f_{0}^{+}$.

Each function $\Phi_{n}(\lambda), n \in \mathbb{N}$, is polynomial in $\lambda$. The function $R(n, m)$ is meromorphic on $\Lambda$ for each $n, m \in \mathbb{Z}$. The singularities of $R(n, m)$ are given by the singularities of

$$
\mathscr{R}_{n}(\lambda)=\frac{f_{n}^{+}(\lambda)}{f_{0}^{+}(\lambda)}=\frac{\tilde{\vartheta}_{n}(\lambda)+m_{+}(\lambda) \tilde{\varphi}_{n}(\lambda)}{f_{0}^{+}(\lambda)} .
$$

The following Lemma follows from Definition 1.

Lemma 8. 1) A real number $\lambda_{0} \in \gamma_{j}^{+}, j=0,1,2$, is a bound state, if the function $\mathscr{R}_{n}(\lambda)$ has a pole at $\lambda_{0}$ for almost all $n \in \mathbb{N}$ (eventually except a finite number of $n$ 's) (it is known that the bound states are simple). 
2) A number $\lambda_{0} \in \Lambda_{-}$, is a resonance, if the function $\mathscr{R}_{n}(\lambda)$ has a pole at $\lambda_{0}$ for almost all $n \in \mathbb{N}$ (eventually except a finite number of $n$ 's). The multiplicity of the resonance is the multiplicity of the pole. If $\operatorname{Re} \lambda_{0}=0$, we call $\lambda_{0}$ antibound state.

3) A real number $\lambda_{0}=\lambda_{0}^{ \pm}$or $\lambda_{0}=\lambda_{1}^{ \pm}$is a virtual state if $\left(\mathscr{R}_{n}(\lambda)\right)^{2}$ or $\mathscr{R}_{n}(\lambda)$ has a pole at $\lambda_{0}$ for almost all $n \in \mathbb{N}$ (eventually except a finite number of $n$ 's).

4) The state $\lambda \in \Lambda$ is a bound state, resonance or virtual state.

We recall that the set of all states of $J$ is denoted by $\mathfrak{S}(J)$.

Each function $f_{n}^{+}(\lambda), n \in \mathbb{N}$, is analytic at all $\lambda \in \Lambda$ whose projection on the complex plane $\mathbb{C}$ is different from $v$. The Jost function $f_{0}^{+}(\lambda)$ has finite number of real zeros on each $\gamma_{k}^{ \pm}$and finite number of complex conjugated zeros on $\Lambda_{-}$.

Remark that if $\lambda_{0} \in \gamma_{k}$, for some $k=0,1,2$ (then $\lambda_{0} \neq \lambda_{0,1}^{ \pm}$), and if $f_{0}^{+}\left(\lambda_{0}\right) \neq 0$ and $\varphi_{2}\left(\lambda_{0}\right) \neq 0$, then the resolvent is analytic at $\lambda_{0}$.

As $\tilde{\vartheta}_{n}(\lambda), \tilde{\varphi}_{n}(\lambda)$ are polynomials then the singularities are zeros of $f_{0}^{+}(\lambda)$ and eventually singularities of $m_{+}$at $\lambda=v$ (as in the unperturbed case).

To describe the states of the general operator $J$ stated in Theorem 3, it is convenient to introduce a special polynomial whose zeros give all states of $J$.

\section{Function $F$ and proofs of main results}

We introduce function $F(\lambda)=\varphi_{2} f_{0}^{+} f_{0}^{-}$.

Lemma 9. Suppose $q_{p} \neq 0$.

i) The function $F(\lambda)=\varphi_{2} f_{0}^{+} f_{0}^{-}(\lambda)$ is polynomial of degree $2 p$ and satisfies

$$
F=\varphi_{2} \tilde{\vartheta}_{0}^{2}+2 \phi \tilde{\vartheta}_{0} \tilde{\varphi}_{0}-\vartheta_{3} \tilde{\varphi}_{0}^{2}=(\lambda-v) \tilde{\vartheta}_{0}^{2}+\frac{1}{a}\left(\lambda^{2}-v^{2}+a^{2}-1\right) \tilde{\vartheta}_{0} \tilde{\varphi}_{0}+(\lambda+v) \tilde{\varphi}_{0}^{2} .
$$

ii) For $\lambda \in \mathbb{C}$, in the limit $|\lambda| \rightarrow \infty$, we have asymptotics

$$
\begin{aligned}
& F=\frac{\lambda^{2 p}}{\left(a_{p} \ldots a_{0}\right)^{2}}\left[-a^{2} q_{p}+\mathcal{O}\left(|\lambda|^{-1}\right)\right], \quad \text { if } p \text { is even, } \\
& F=\frac{\lambda^{2 p}}{\left(a_{p} \ldots a_{0}\right)^{2}}\left[-q_{p}+\mathcal{O}\left(|\lambda|^{-1}\right)\right], \quad \text { if } p \text { is odd, }
\end{aligned}
$$

where $\mathcal{O}\left(|\lambda|^{-1}\right)$ is uniformly bounded in a. In particular, if $\lambda \in \mathbb{R}$ and $|\lambda| \rightarrow \infty$, we have $\operatorname{sign}(F)=-\operatorname{sign}\left(q_{p}\right)$.

iii) In the limit $a \rightarrow 0$, the function $F$ behaves as follows:

if $p$ is even, then

$$
\begin{aligned}
F= & \frac{1}{a^{p}}\left[\left(\lambda-\tilde{v}_{p-1}\right)\left(\lambda-\tilde{v}_{p}\right)-1\right]\left[\left(\lambda-\tilde{v}_{p-1}\right)\left(1-q_{p}(\lambda-v)\right)-(\lambda-v)\right] \\
& \cdot \prod_{k=1}^{p-3}\left[\left(\lambda-\tilde{v}_{k}\right)\left(\lambda-\tilde{v}_{k+1}\right)-1\right]^{2}+\mathcal{O}\left(a^{2-p}\right)
\end{aligned}
$$


if $p$ is odd

$$
F=\frac{-q_{p}}{a^{p+1}}\left[\left(\lambda-\tilde{v}_{p}\right)(\lambda+v)-1\right] \prod_{k=1}^{p-2}\left[\left(\lambda-\tilde{v}_{k}\right)\left(\lambda-\tilde{v}_{k+1}\right)-1\right]^{2}+\mathcal{O}\left(a^{1-p}\right) .
$$

Here $\mathcal{O}\left(a^{j}\right)$ is uniformly bounded in $\lambda \in \mathbb{C}$.

iv) Put $q_{j}=t q_{j}^{0}$ for all $q_{j}^{0} \neq 0$ fixed and $t \rightarrow \infty$. Then,

$$
\begin{aligned}
& F(\lambda)=t^{2 p} \frac{(\lambda-v)}{a^{p}}\left[\prod_{k=1}^{p}\left(q_{p}^{0}\right)^{2}+\mathcal{O}\left(t^{2 p-1}\right)\right], \text { if } p \text { is even } \\
& F(\lambda)=t^{2 p} \frac{(\lambda+v)}{a^{p+1}}\left[\prod_{k=1}^{p}\left(q_{p}^{0}\right)^{2}+\mathcal{O}\left(t^{2 p-1}\right)\right], \text { if } p \text { is odd },
\end{aligned}
$$

uniformly bounded in $\lambda \in \mathbb{C}$.

Proof: We have

$$
\begin{aligned}
f_{0}^{+} f_{0}^{-} & =\left(\tilde{\vartheta}_{0}+m_{+} \tilde{\varphi}_{0}\right)\left(\tilde{\vartheta}_{0}+m_{-} \tilde{\varphi}_{0}\right)=\tilde{\vartheta}_{0}^{2}+\left(m_{+}+m_{-}\right) \tilde{\vartheta}_{0} \tilde{\varphi}_{0}+m_{+} m_{-} \tilde{\varphi}_{0}^{2} \\
& =\tilde{\vartheta}_{0}^{2}+\frac{2 \phi}{\varphi_{2}} \tilde{\vartheta}_{0} \tilde{\varphi}_{0}+\frac{\phi^{2}+1-\Delta^{2}}{\varphi_{2}^{2}} \tilde{\varphi}_{0}^{2}=\tilde{\vartheta}_{0}^{2}+\frac{2 \phi}{\varphi_{2}} \tilde{\vartheta}_{0} \tilde{\varphi}_{0}+\frac{-\vartheta_{3} \varphi_{2}}{\varphi_{2}^{2}} \tilde{\varphi}_{0}^{2} \\
& =\tilde{\vartheta}_{0}^{2}+\frac{2 \phi}{\varphi_{2}} \tilde{\vartheta}_{0} \tilde{\varphi}_{0}-\frac{\vartheta_{3}}{\varphi_{2}} \tilde{\varphi}_{0}^{2},
\end{aligned}
$$

where we have used (2.11) and (2.15). The degree $2 p$ will come from $\varphi_{2}=\lambda-v$ and asymptotics (4.2), (4.3).

Now as $F$ is polynomial, in order to prove the asymptotics $|\lambda| \rightarrow \infty$ on $\mathbb{C}$ it is enough to consider $\lambda \rightarrow+\infty$ on $\gamma_{2}^{+}, a \rightarrow 0$ or $q_{j} \rightarrow \infty$ for $\lambda \in \gamma_{1}^{+}$. The proof thus follows from the asymptotics of the Jost functions given in Lemma 7.

From iii), Lemma 9, we get the leading orders of the zeros of $F$ as $a \rightarrow 0$ which correspond to the leading orders of the states. Using Lemma 7 we know if the limiting state is a bound state or a resonance. Recall the eigenvalues of the matrix (1.7) given by $z_{n, \pm}=v_{n}^{+} \pm\left|v_{n}^{-2}+1\right|^{\frac{1}{2}}$ (see (1.8)). If $q_{p} \neq 0$ and

if $p$ is even, then there are at most $p$ eigenvalues $z_{n, \pm}, n=1,2, \ldots, \frac{p}{2}$, where

$$
v_{n}^{+}=\frac{\tilde{v}_{2 n-1}+\tilde{v}_{2 n}}{2}=\frac{q_{2 n-1}+q_{2 n}}{2}, \quad v_{n}^{-}=\frac{\tilde{v}_{2 n-1}-\tilde{v}_{2 n}}{2}=v+\frac{q_{2 n-1}-q_{2 n}}{2},
$$

if $p$ is odd, then there are at most $p+1$ eigenvalues $z_{n, \pm}, n=1,2, \ldots, \frac{(p-1)}{2}$, where

$$
\begin{gathered}
v_{n}^{+}=\frac{\tilde{v}_{2 n-1}+\tilde{v}_{2 n}}{2}=\frac{q_{2 n-1}+q_{2 n}}{2}, v_{n}^{-}=\frac{\tilde{v}_{2 n-1}-\tilde{v}_{2 n}}{2}=v+\frac{q_{2 n-1}-q_{2 n}}{2}, \\
v_{(p+1) / 2}^{+}=\frac{\tilde{v}_{p}-v}{2}=\frac{q_{p}}{2}, v_{(p+1) / 2}^{-}=\frac{\tilde{v}_{p}+v}{2}=v+\frac{q_{p}}{2} .
\end{gathered}
$$


Recall that, as perturbations have finite support, then there are also two flat bands (two eigenvalues with infinite multiplicities) given by $z_{n, \pm}= \pm\left|v^{2}+1\right|^{\frac{1}{2}}$, where $n \geqslant \frac{p}{2}+1$ if $p$ is even or $n \geqslant \frac{(p+1)}{2}+1$ if $p$ is odd. Similar, using Lemma 7, we get the leading orders of the resonances. In the even case the resonances can converge to complex number - zeros of the factor $\left(\lambda-\tilde{v}_{p-1}\right)\left\{\left(\lambda-\tilde{v}_{p}\right)-\frac{2 \delta}{\lambda-v}\right\}-$ 1 in (3.4) or equivalently zeros of the polynomial $\left(\lambda-\tilde{v}_{p-1}\right)\left(1-q_{p}(\lambda-v)\right)-(\lambda-v)$ (see (4.4)).

This implies Theorem 4.

Theorem 5 follows from 7 and iv) in Lemma 9.

In the next Lemma we state the crucial properties of the function $F$.

Lemma 10. i) Suppose that $\lambda_{1} \in \gamma_{k}^{+}$, for $k=0,1$ or 2 , and either

a) $f_{0}^{+}\left(\lambda_{1}\right)=0$, i.e. $\lambda_{1}$ is an eigenvalue of $J$ with the eigenfunction $y_{n}=f_{n}^{+}\left(\lambda_{1}\right)$, or

b) $\lambda_{1}=v$. Let $\lambda_{1}$ also denote the projection of $\lambda_{1} \in \lambda_{k}^{+}$on $\mathbb{C}$.

Then $(-1)^{k} \dot{F}\left(\lambda_{1}\right)<0$ and function $F$ has simple zeros at all bound states of J. Moreover if $\lambda_{1}=v$, then $\tilde{\varphi}_{0}(v)=0, f_{0}^{+}(\lambda)$ is analytic at $\lambda=v \in \gamma_{1}^{+}$and $f_{0}^{+} \neq 0$.

ii) We have $F(\lambda)=$

$$
\varphi_{2}\left(\tilde{\vartheta}_{0}+\frac{\phi}{\varphi_{2}} \tilde{\varphi}_{0}\right)^{2}+\frac{1-\Delta^{2}}{\varphi_{2}} \tilde{\varphi}_{0}^{2}=\varphi_{2}\left(\tilde{\vartheta}_{0}+\frac{\phi}{\varphi_{2}} \tilde{\varphi}_{0}\right)^{2}+\frac{-\left(\lambda^{2}-\lambda_{0}^{2}\right)\left(\lambda^{2}-\lambda_{1}^{2}\right)}{4 a^{2} \varphi_{2}} \tilde{\varphi}_{0}^{2},
$$

where $\lambda_{0}=\lambda_{0}^{\mp}$ and $\lambda_{1}=\lambda_{1}^{ \pm}$are the endpoints of $\sigma_{\mathrm{ac}}\left(J^{0}\right)=\left[\lambda_{0}^{+}, \lambda_{1}^{-}\right] \cup\left[\lambda_{1}^{+}, \lambda_{0}^{-}\right]$.

We have $F(\lambda)<0$, for $\lambda \in\left(\lambda_{0}^{+}, \lambda_{1}^{-}\right)$, and $F(\lambda)>0$, for $\lambda \in\left(\lambda_{1}^{+}, \lambda_{0}^{-}\right)$.

Remarks. 1) Lemma 10 (with proper modifications) is also true for general Jacobi operators on the half-line and is proven in paper [4]. The methods remind the approach of [7] to the periodic Schrödinger operator plus compactly supported potentials on the half-line.

2) It follows that $F(\lambda)$, which is real on the real axis, is decreasing function at any eigenvalue $\lambda_{1} \in \gamma_{0,2}^{+}$, and increasing function at any eigenvalue $\lambda_{1} \in \gamma_{1}^{+}$.

It follows that all bound states of $J$ are simple and that for any two eigenvalues $\lambda_{1,2} \in \gamma_{k}^{+}$ such that the interval $\Omega^{+}=\left(\lambda_{1}, \lambda_{2}\right) \subset \gamma_{k}^{+}$does not contain any other bound states there is an odd number of antibound states $\lambda_{0}$ in the same interval $\Omega^{-} \subset \gamma_{k}^{-} \subset \Lambda_{-}$on the second sheet, and $(-1)^{k} \dot{F}\left(\lambda_{0}\right)>0$.

3) From ii) it follows that there is always at least one eigenvalue in the middle gap $\gamma_{1}$ or a virtual state at $\lambda_{1}^{ \pm}$. Moreover, using that from Lemma 9 it follows that function $F$ has the same sign when $\lambda \rightarrow \pm \infty$, we get that

if $\operatorname{sign}(F)( \pm \infty)<0$, then there are at least two eigenvalues: one in $\gamma_{1}$ another in $\gamma_{2}$ (which can become virtual states); if $\operatorname{sign}(F)( \pm \infty)>0$ and $0<a<1$, then there are at least two eigenvalues: one in $\gamma_{0}$, another in $\gamma_{1}$ (which can become virtual states).

Now the proof of Theorem 2 follows from the following two Lemmata which are proved in [4] in the general case. 

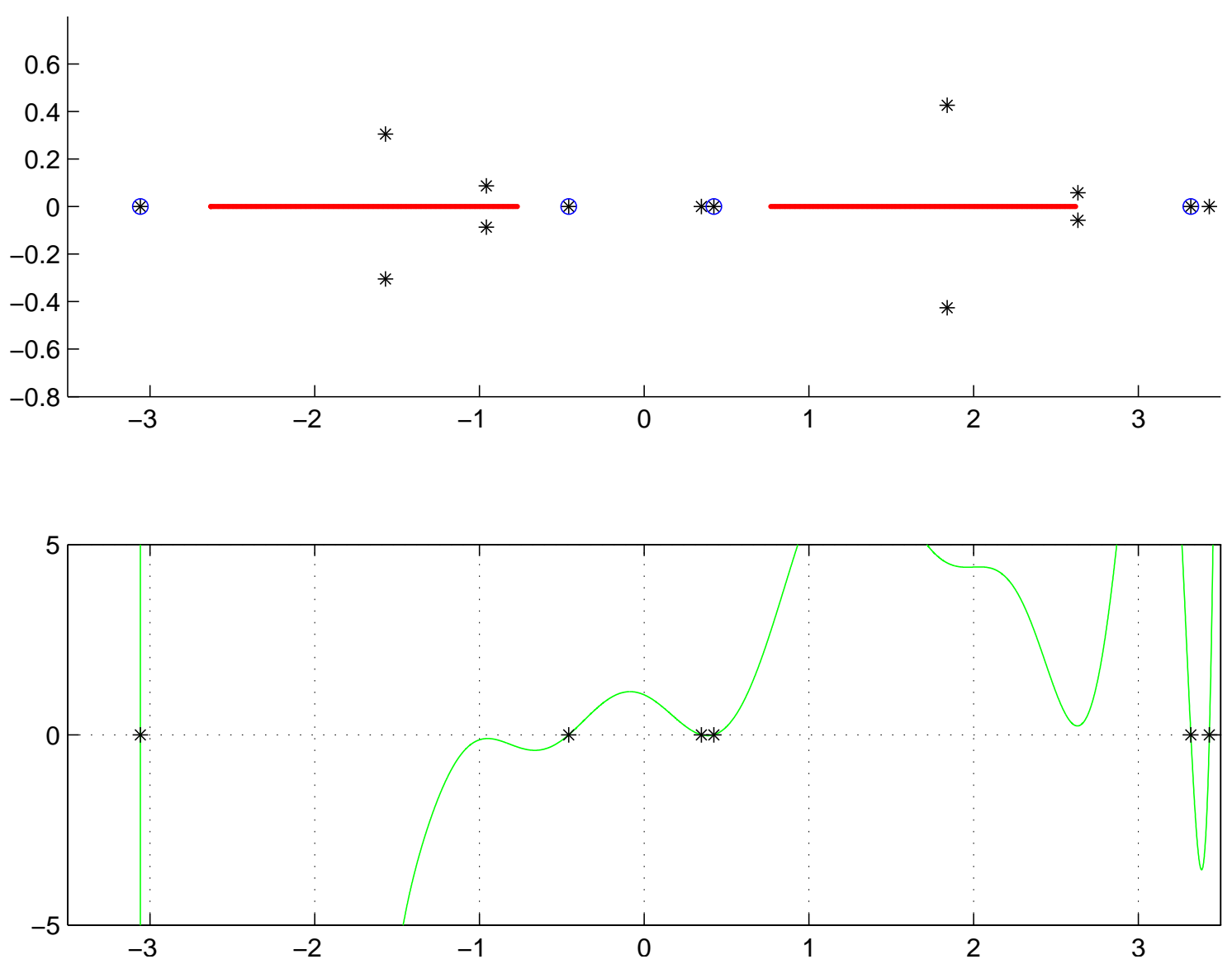

Fig 3. Function $F$ and the states for $p=7$, the bound states are encircled.

Lemma 11 (Virtual states). Let $\lambda_{0}$ denote any of $\lambda_{0,1}^{ \pm}$and let $\lambda=\lambda_{0}+\epsilon$ for $\epsilon>0$ small enough. i) If $\lambda, \lambda_{0} \neq v$ and $f_{0}^{+}\left(\lambda_{0}\right)=0$, then $\lambda_{0}$ is a simple zero of $F, \lambda_{0}$ is a virtual state of $J$, and

$$
f_{0}^{+}(\lambda)=\tilde{\varphi}_{0}\left(\lambda_{0}\right) c \sqrt{\epsilon}+\mathcal{O}(\epsilon), \mathscr{R}_{n}(\lambda)=\frac{f_{n}^{+}(\lambda)}{\tilde{\varphi}_{0}\left(\lambda_{0}\right) c \sqrt{\epsilon}}(1+\mathcal{O}(\sqrt{\epsilon})), c \tilde{\varphi}_{0}\left(\lambda_{0}\right) \neq 0 .
$$

ii) If $\lambda_{0}=v$ (which happens if $a=1$ ) and $\tilde{\varphi}_{0}\left(\lambda_{0}\right) \neq 0$, then $F\left(\lambda_{0}\right) \neq 0$ and each $\mathscr{R}_{n}(),. n \in \mathbb{N}$, does not have singularity at $\lambda_{0}$ and $\lambda_{0}$ is not a virtual state of $J$.

iii) If $\lambda_{0}=v$ and $\tilde{\varphi}_{0}\left(\lambda_{0}\right)=0$, then $\lambda_{0}$ is a virtual state of $J, f_{0}^{ \pm}\left(\lambda_{0}\right) \neq 0, \lambda_{0}$ is simple zero of $F$, and each $\left(\mathscr{R}_{n}(.)\right)^{2}, n \in \mathbb{N}$, has pole at $\lambda_{0}$.

Lemma 12. The projection $\pi: \Lambda \mapsto \mathbb{C}$ of the set of states of $J$ on $\Lambda$ coincides with the set of zeros of $F$ on the complex plane $\mathbb{C}$ :

$$
\pi \mathfrak{S}(J)=\operatorname{Zeros}(F)
$$

Moreover, the multiplicities of bound states and resonances are equal to the multiplicities of zeros of F. All bound states are simple. 
Suppose $\lambda_{0}=\lambda_{0}^{ \pm}$or $\lambda_{0}=\lambda_{1}^{ \pm}$and $\tilde{\varphi}_{0}\left(\lambda_{0}\right) \neq 0$. Then $\lambda_{0}$ is a virtual state iff $F(\lambda)$ has zero at $\lambda_{0}$. It will be automatically simple.

In the next Lemma we consider a special case when we have a simple criterium when $\lambda=v$ is a state.

Lemma 13. Suppose that $p$ is even and for any $n \in \mathbb{N}, \tilde{v}_{2 n+1}=v_{2 n+1}=v$.

Then $\tilde{\varphi}_{0}(v)=0$ and $F(v)=0$. Thus $\lambda=v$ is a state.

Proof: From the well known explicit formula $\varphi_{2 n}=(\lambda-v) \sin n 2 \varkappa / \sin 2 \varkappa$ it follows that $\varphi_{2 n}(v)=0$ for any $n \in \mathbb{N}$. From the equation $J y=\lambda y$ we have the iteration formula:

$$
\tilde{\varphi}_{2 n}=\frac{\left(\lambda-\tilde{v}_{2 n+1}\right) \tilde{\varphi}_{2 n+1}-\tilde{\varphi}_{2 n+2}}{a} .
$$

But $\tilde{\varphi}_{k}=\varphi_{k}$ for $k \geqslant p+1$ and $\tilde{v}_{2 n+1}=v$. Thus, starting with $2 n=p$ and iterating (4.8), we get $\tilde{\varphi}_{2 n}(v)=0, \tilde{\varphi}_{2 n-2}(v)=0, \ldots, \tilde{\varphi}_{0}(v)=0$ : all functions $\tilde{\varphi}_{k}$ with even indexes are zeros at $\lambda=v$. Then from (4.1) it follows that $F(v)=0$.

\section{Examples $p=1$ and $p=2$.}

In this Section we consider the special cases $p=1$ and $p=2$ when the properties of the states can be analyzed in more details. Using that

$$
\begin{gathered}
f_{p}^{+}=\frac{\left(\lambda-\tilde{v}_{p+1}\right) f_{p+1}^{+}-a_{p+1} f_{p+2}^{+}}{a_{p}}=\frac{\xi_{+}^{p+1}}{a_{p}}\left((\lambda+v)-a m_{+}\right)=\frac{\xi_{+}^{p+1}}{a_{p}} \frac{1+a \xi_{-}^{2}}{\lambda-v}, \\
f_{p-1}^{+}=\frac{\left(\lambda-\tilde{v}_{p}\right) f_{p}^{+}-a_{p} f_{p+1}^{+}}{a_{p-1}}=\frac{\xi^{p+1}}{a_{p} a_{p-1}}\left(\left(\lambda-\tilde{v}_{p}\right) \frac{1+a \xi_{-}^{2}}{\lambda-v}-a_{p}^{2}\right)
\end{gathered}
$$

and $\varphi_{2}=\lambda-v, \xi_{+}^{2} \xi_{-}^{2}=1, \xi_{+}^{2}+\xi_{-}^{2}=2 \Delta=\left(\lambda^{2}-v^{2}-a^{2}-1\right) / a \Rightarrow 2 a \Delta+1+a^{2}=\lambda^{2}-v^{2}$, we get for $p=1, a_{0}=a, a_{1}=1, v_{1}=v+q_{1}$,

$$
F(\lambda)=\varphi_{2} f_{0}^{+} f_{0}^{-}=a^{-2}\left(-q_{1} \lambda^{2}+\lambda\left[q_{1}^{2}+a^{2}\right]+\left(q_{1}^{2} v+q_{1}\left(v^{2}+1-a\right)-v a^{2}\right)\right),
$$

If $v=v_{1}$ (the unperturbed case) then $F=a^{2}(\lambda-v)$ and $\lambda=v$ is the only state, see Lemma 6 . The discriminant of the quadratic equation is

$$
D=\left(a^{2}-\left(v_{1}^{2}-v^{2}\right)\right)^{2}+4\left(v_{1}-v\right)^{2}=\left(q_{1}^{2}+2 v q_{1}-a^{2}\right)^{2}+4 q_{1}^{2}>0 \text { if } v_{1} \neq v .
$$

Thus we get that the states are real. By Lemma 10 on $F$, part ii), both states are bound states: no resonances for $p=1$. One can check directly that if perturbation is non-trivial $\left(v \neq v_{1}\right)$ then there are no virtual states if $p=1$ : if $\lambda_{0}=\lambda_{0}^{ \pm}$is virtual state then $\xi_{-}^{2}=\Delta\left(\lambda_{0}\right)= \pm 1$, and

$$
f_{0}^{+}\left(\lambda_{0}\right)=\frac{ \pm 1}{a}\left(\left(\lambda-v_{1}\right) \frac{1 \pm a}{\lambda-v}-1\right)=0 \Leftrightarrow\left(\lambda_{0}-v_{1}\right)(1 \pm a)=\lambda_{0}-v, \lambda_{0} \neq v,
$$

which never happens. Thus we have 
Proposition 14. For $p=1, v \neq v_{1}$, J has two real bound states:

$$
\lambda_{ \pm}=\frac{\left[q_{1}^{2}+a^{2}\right] \pm \sqrt{\left(q_{1}^{2}+2 v q_{1}-a^{2}\right)^{2}+4 q_{1}^{2}}}{2 q_{1}}=\frac{q_{1}}{2}+\frac{a^{2}}{2 q_{1}} \pm \sqrt{\left(\frac{q_{1}}{2}+v-\frac{a^{2}}{2 q_{1}}\right)^{2}+1}
$$

In the limit $a \rightarrow 0$, we get straightforward $\lambda_{1,2}=\frac{1}{2}\left(q_{1} \pm \sqrt{\left(2 v+q_{1}\right)^{2}+4}\right)+\mathcal{O}\left(a^{2}\right)$. As $v \rightarrow \infty$, we have $\lambda_{1,2} \sim \pm v \rightarrow \pm \infty$. As $v \rightarrow 0$, we have $\lambda_{1,2} \rightarrow\left(2 q_{1}\right)^{-1}\left(\left[q_{1}^{2}+a^{2}\right] \pm \sqrt{\left(q_{1}^{2}-a^{2}\right)^{2}+4 q_{1}^{2}}\right)$. Next we get:

if $q_{1} \rightarrow 0$, then $\lambda_{+} \sim a^{2} / q_{1} \rightarrow \infty$, and $\lambda_{-} \rightarrow v$;

if $q_{1} \rightarrow \infty$, we have $\lambda_{+} \sim q_{1} \rightarrow \infty$, and $\lambda_{-} \rightarrow-v$.

Now we consider in detail the properties of the states in the simplest non-trivial case $p=2$, which allows the complex resonances. Let $D\left(p_{3}\right)$ denote the generalized discriminant of a special cubic polynomial which will be explained below and given by the following cumbersome formula:

$$
\begin{aligned}
D\left(p_{3}\right)= & \left(v q_{2}+q_{2}^{2}\right)^{2} q_{2}^{2}\left(2 v q_{2}-v^{2}-a^{2}-1\right)^{2}-4\left(v q_{2}+q_{2}^{2}\right)^{3}\left\{\left(v q_{2}-v^{2}-1\right)\left(v q_{2}-a^{2}\right)-v^{2} a^{2}\right\}- \\
& -4 q_{2}^{4}\left(2 v q_{2}-v^{2}-a^{2}-1\right)^{3}+ \\
& +18 q_{2}\left(v q_{2}+q_{2}^{2}\right) q_{2}\left(2 v q_{2}-v^{2}-a^{2}-1\right)\left\{\left(v q_{2}-v^{2}-1\right)\left(v q_{2}-a^{2}\right)-v^{2} a^{2}\right\}- \\
& -27 q_{2}^{2}\left\{\left(v q_{2}-v^{2}-1\right)\left(v q_{2}-a^{2}\right)-v^{2} a^{2}\right\}^{2} .
\end{aligned}
$$

Proposition 15. $i)$ Suppose $p=2$ and $q_{2} \neq 0$. Then $J$ has always two bound states and two resonances (or virtual states). In the limit $a \rightarrow 0+$, the bound states converge to

$$
\lambda_{1,2}^{0}= \pm \sqrt{\left(v+\frac{q_{1}-q_{2}}{2}\right)^{2}+1}+\frac{q_{1}+q_{2}}{2}
$$

and the resonances converge to

$$
\lambda_{3,4}^{0}=v+\frac{q_{1}}{2} \pm \sqrt{\frac{q_{1}\left(q_{2} q_{1}-4\right)}{4 q_{2}}} .
$$

Suppose that $\tilde{v}_{1}=v$. Then $\lambda=v$ is always a state.

Moreover, let $D\left(p_{3}\right)$ denote the generalized discriminant given by Formula (5.1). Then, all four states of $J$ are real iff $D\left(p_{3}\right)>0$. If $D\left(p_{3}\right)<0$, then there are always two complex conjugated resonances.

ii) Suppose $p=2, \tilde{v}_{1}=v$. We have the following asymptotic properties of the states:

1) for $q_{2}$ small enough, $J$ has precisely two non-real complex conjugated resonances;

2 ) in the limit $q_{2} \rightarrow \infty$, the states of J either go to infinity or converge to the real state $\lambda=v$;

$3)$ in the limit $v \rightarrow \infty$, the states are of order $|v|$. Moreover, let $\mu_{1,2,3}$ denote the zeros of $\mu^{3}-\mu^{2}-$ $\mu+q_{2}=0$, which are real if

$$
q_{2} \in\left[\frac{11-\sqrt{11^{2}+5 \cdot 27}}{27}, \frac{11+\sqrt{11^{2}+5 \cdot 27}}{27}\right]
$$


and contain one complex conjugate pair otherwise. Then $\lambda_{1,2,3} / v \rightarrow \mu_{1,2,3}$ in the limit $\left.v \rightarrow \infty ; 4\right)$ for $v$ small enough we do not have non-numerical results;

5 ) in the limit $a \rightarrow 0+$, the two resonances converge to $\lambda=v$.

Some of this results can be generalized to any $p$ (see Theorem 5). The fact that $\lambda=v$ is always a state in the special case $p=2, \tilde{v}_{1}=v$, can be generalized to any even $p$ (see Lemma 13). We proceed now to the proofs.

Proof: From the properties of function $F$ we know that $J$ has always at least two bound states (or eventually virtual states).

For $p=2$ we have

$$
f_{0}^{+}(\lambda)=\frac{\xi_{+}^{2}}{a^{2}}\left(\left(\lambda-v_{1}\right)\left(\left(\lambda-v_{2}\right) a-a^{2} m_{+}\right)-a\right), f_{0}^{-}(\lambda)=\overline{f_{0}^{+}(\bar{\lambda})} .
$$

We get $F=\varphi_{2} f_{0}^{+} f_{0}^{-}=$

$$
\begin{array}{r}
=\frac{1}{a^{2}}\left[(\lambda-v)\left\{\left(\lambda-v_{1}\right)\left(\lambda-v_{2}\right)-1\right\}^{2}-\left\{\left(\lambda-v_{1}\right)\left(\lambda-v_{2}\right)-1\right\}\right. \\
\left(\lambda-v_{1}\right)\left(\lambda^{2}-v^{2}+a^{2}-1\right)+ \\
\left.+\left(\lambda-v_{1}\right)^{2} a^{2}(\lambda+v)\right],
\end{array}
$$

where we used that $m_{+}+m_{-}=2 \Phi / \varphi_{2}$ and $m_{+} m_{-}=-\vartheta_{3} / \varphi_{2}$, where $\varphi_{2}=\lambda-v, \Phi=$ $\left(\lambda^{2}-v^{2}+a^{2}-1\right) / 2 a, \vartheta_{3}=-\lambda-v$.

Suppose $v_{1}=v$ and $\lambda \neq v$. From (5.5) we get $\frac{a^{2} F(\lambda)}{\lambda-v}=$

$$
\begin{aligned}
& =-\left(\lambda^{2}-v^{2}-a^{2}-1\right)(\lambda-v) q_{2}+(\lambda-v)^{2} q_{2}^{2}+a^{2}= \\
& =-q_{2} \lambda^{3}+\lambda^{2}\left(v q_{2}+q_{2}^{2}\right)-\lambda q_{2}\left(2 v q_{2}-v^{2}-a^{2}-1\right)+\left(v q_{2}-v^{2}-1\right)\left(v q_{2}-a^{2}\right)-v^{2} a^{2}
\end{aligned}
$$

As $F(\lambda)=\frac{\lambda-v}{a^{2}} p_{3}(\lambda)$ we have: $\operatorname{sign} F=-\operatorname{sign} q_{2}$ in the limit $\lambda \rightarrow \pm \infty$. As $F$ is strictly negative under the first band and strictly positive over the second band, we have:

if $q_{2}<0$ then there is one bound state in $\gamma_{0}^{+}$; if $q_{2}>0$ then there is one bound state in $\gamma_{2}^{+}$;

state $\lambda=v \in \gamma_{1}$;

the other 2 states are either real, then they belong to the same gap, or complex conjugate.

The right hand side of (5.7) is the cubic polynomial with real coefficients in $\lambda$ and can have 3 real zeros or one real zero and two complex conjugated zeros. Denote the respective coefficients in (5.7) by $k_{0}, k_{1}, k_{2}, k_{3}$, then we have

$$
\begin{aligned}
& \frac{a^{2} F(\lambda)}{\lambda-v}=p_{3}(\lambda)=k_{0} \lambda^{3}+k_{1} \lambda^{2}+k_{2} \lambda+k_{3}, \\
& k_{0}=-q_{2}, \quad k_{1}=v q_{2}+q_{2}^{2}, k_{2}=-q_{2}\left(2 v q_{2}-v^{2}-a^{2}-1\right), k_{3}=\left(v q_{2}-v^{2}-1\right)\left(v q_{2}-a^{2}\right)-v^{2} a^{2} .
\end{aligned}
$$

Remark 3.104 on page 127 from [21], states that if the generalized discriminant of $p_{3}$

$$
D\left(p_{3}\right)=k_{1}^{2} k_{2}^{2}-4 k_{1}^{3} k_{3}-4 k_{0} k_{2}^{3}+18 k_{0} k_{1} k_{2} k_{3}-27 k_{0}^{2} k_{3}^{2}
$$


is strictly positive then all the zeros of (5.7) are real and disctinct. If $D\left(p_{3}\right)<0$, then there are two complex conjugated zeros. The discriminant $D\left(p_{3}\right)$ is given in (5.1).

Thus we have proven the first part i) of Proposition 15 except asymptotics (5.2) and (5.3 which we postpone to the end of this section.

Suppose $q_{2} \rightarrow 0$. Then

$$
k_{0}=-q_{2}, k_{1}=v q_{2}+\mathcal{O}\left(q_{2}^{2}\right), k_{2}=q_{2}\left(v^{2}+a^{2}+1\right)+\mathcal{O}\left(q_{2}^{2}\right), k_{3}=a^{2}+\mathcal{O}\left(q_{2}\right) .
$$

Then $D=-27 k_{0}^{2} k_{3}^{2}+\mathcal{O}\left(q_{2}^{3}\right)=-27 q_{2}^{2} a^{4}+\mathcal{O}\left(q_{2}^{3}\right)$ which implies that there are two non-real resonances.

Suppose $q_{2} \rightarrow \infty$. Then directly from the equation

$$
-q_{2} \lambda^{3}+q_{2}^{2} \lambda^{2}-2 v q_{2}^{2} \lambda+v^{2} q_{2}^{2}=\mathcal{O}\left(q_{2}\right) \Leftrightarrow \lambda^{2}-2 v \lambda+v^{2}=(\lambda-v)^{2}=\mathcal{O}\left(\lambda^{3} q_{2}^{-1}\right), q_{2} \rightarrow \infty,
$$

we get that the states which remain bounded as $q_{2} \rightarrow \infty$ converge to $v$.

Suppose $v \rightarrow \infty$. Then the equation is $-q_{2} \lambda^{3}+v q_{2} \lambda^{2}+q_{2} v^{2} \lambda-v^{3} q_{2}^{2}=\mathcal{O}\left(v^{2}\right)$. Put $\lambda=v \mu$, then $-q_{2} v^{3}\left(\mu^{3}-\mu_{2}-\mu+q_{2}\right)=\mathcal{O}\left(v^{2}\right) \Leftrightarrow \mu^{3}-\mu^{2}-\mu+q_{2}=\mathcal{O}\left(\left(v q_{2}\right)^{-1}\right), v \rightarrow \infty$. The equation $\mu^{3}-\mu^{2}-\mu+q_{2}=0$ has the generalized discriminant $D=1+4 q_{2}+4+16 q_{2}-27 q_{2}^{2}=$ $-27 q_{2}^{2}+22 q_{2}+5$, whose zeros are

$$
x_{ \pm}=\frac{11 \pm \sqrt{11^{2}+5 \cdot 27}}{27} .
$$

Denote $\mu_{1,2,3}$ the zeros of the equation $\mu^{3}-\mu^{2}-\mu+q_{2}=0$. We proved that, as $v \rightarrow \infty$, the states $\lambda_{1,2,3}=\mathcal{O}(v)$, moreover $\lambda_{1,2,3} / v$ converge to the zeros of the equation $\mu^{3}-\mu^{2}-\mu+q_{2}=0$, which are real if $q_{2} \in\left[x_{-}, x_{+}\right]$. If $q_{2}<x_{-}$or $q_{2}>x_{+}$then the two zeros are complex conjugated. Thus we have proven the part ii) of Proposition 15. In the case $v \rightarrow 0$, the equation does not simplify.

Asymptotics (5.2) and (5.3) as $a \rightarrow 0$. As a special case of the asymptotics in Lemma 7, we get, for $\lambda \in \gamma_{1}^{+}$,

$$
\begin{aligned}
f_{0}^{+} & =\frac{1}{a^{2}}\left(\frac{a}{2 \delta}+\mathcal{O}\left(a^{3}\right)\right)\left(\left(\lambda-v_{1}\right)\left[\left(\lambda-v_{2}\right) a+\mathcal{O}\left(a^{3}\right)\right]-a\right)= \\
& =\frac{1}{a^{2}}\left(\frac{\left(\lambda-v_{1}\right)\left(\lambda-v_{2}\right)-1}{2 \delta} a^{2}+\mathcal{O}\left(a^{4}\right)\right)=\frac{\left(\lambda-v_{1}\right)\left(\lambda-v_{2}\right)-1}{\lambda^{2}-v^{2}-1}+\mathcal{O}\left(a^{2}\right) .
\end{aligned}
$$

Thus the bound states in $\gamma_{1}^{+}$which are solutions of $f_{0}^{+}(\lambda)=0$ in the limit $a \rightarrow 0$ are asymptotically solutions of the equation

$$
\frac{\left(\lambda-v_{1}\right)\left(\lambda-v_{2}\right)-1}{\lambda^{2}-v^{2}-1}=0 \Leftrightarrow \lambda^{2}-\left(v_{1}+v_{2}\right) \lambda+v_{1} v_{2}-1=0
$$

if $\lambda^{2} \neq v^{2}+1$ (which happens if $\lambda$ is a virtual state). We get two solutions $\left(\tilde{v}_{1}=v+q_{1}\right.$, $\left.\tilde{v}_{2}=-v+q_{2}\right)$

$$
z_{1, \pm}=\frac{\tilde{v}_{1}+\tilde{v}_{2} \pm \sqrt{\left(\tilde{v}_{1}-\tilde{v}_{2}\right)^{2}+4}}{2}= \pm \sqrt{\left(v+\frac{q_{1}-q_{2}}{2}\right)^{2}+1} \pm \frac{q_{1}+q_{2}}{2}
$$


which are the leading terms in the expansion of the bound states in $\gamma_{1}^{+}$as $a \rightarrow 0$ (see Theorem 4).

Similarly we get the resonances in $\gamma_{1}^{-}$and states in $\gamma_{0,2}^{ \pm}$in the limit $a \rightarrow 0$. The resonances in $\gamma_{1}^{-}$are formally also zeros of $f_{0}^{-}(\lambda)$ in $\gamma_{1}^{+}$which, if $v_{1} \neq v$, in the leading order $a^{-2}$ are solutions of the equation $\left(v_{2}+v\right) \lambda^{2}-\left(v_{2} v+v^{2}+v_{1}\left(v_{2}+v\right)\right) \lambda+v_{1}\left(v_{2} v+v^{2}+1\right)-v=0$ with zeros given in (1.11):

$$
\mu_{1,2}^{0}=\frac{q_{2}\left(2 v+q_{1}\right) \pm \sqrt{q_{2} q_{1}\left(q_{2} q_{1}-4\right)}}{2 q_{2}}=v+\frac{q_{1}}{2} \pm \sqrt{\frac{q_{1}\left(q_{2} q_{1}-4\right)}{4 q_{2}}}
$$

which can be real antibound states or complex conjugated resonances. If $v_{1}=v$ then the leading order as $a \rightarrow 0$ of the antibound state is $\lambda=v$.

Suppose now that $\lambda_{0}$ is a real double root: $F\left(\lambda_{0}\right)=0, \dot{F}\left(\lambda_{0}\right)=0$. Suppose $\lambda_{0} \neq v$. Using the identity

$$
a^{2} \dot{F}(\lambda)=a^{2} \frac{F(\lambda)}{\lambda-v}+(\lambda-v) \frac{\partial}{\partial \lambda}\left(a^{2} \frac{F(\lambda)}{\lambda-v}\right)
$$

we have at $\lambda=\lambda_{0}$ :

$$
a^{2} \dot{F}\left(\lambda_{0}\right)=\left(\lambda_{0}-v\right) \frac{\partial}{\partial \lambda}\left(a^{2} \frac{F(\lambda)}{\lambda-v}\right)_{\mid \lambda=\lambda_{0}}=0 .
$$

In the special case $p=2, v_{1}=v$, we get using (5.7):

$$
\frac{\partial}{\partial \lambda}\left(a^{2} \frac{F(\lambda)}{\lambda-v}\right)_{\mid \lambda=\lambda_{0}}=-3 q_{2} \lambda_{0}^{2}+2 q_{2}\left(v+q_{2}\right) \lambda_{0}-q_{2}\left(2 v q_{2}-v^{2}-a^{2}-1\right)=0 .
$$

It follows that if $F\left(\lambda_{0}\right)=\dot{F}\left(\lambda_{0}\right)=0$ and $\lambda_{0} \neq v$ then $\lambda_{0}$ is a zero of the quadratic equation $3 \lambda^{2}-2\left(v+q_{2}\right) \lambda+\left(2 v q_{2}-v^{2}-a^{2}-1\right)=0$ :

$$
\lambda_{0}=\frac{\left(v+q_{2}\right) \pm \sqrt{\left(v+q_{2}\right)^{2}-3\left(2 v q_{2}-v^{2}-a^{2}-1\right)}}{3}=\frac{\left(v+q_{2}\right) \pm \sqrt{\left(2 v-q_{2}\right)^{2}+3\left(a^{2}+1\right)}}{3} .
$$

The state has multiplicity 2 and necessarily are the antibound states as all bound states are simple. Thus we get

Proposition 16 (Double antibound state). Suppose $p=2, v_{1}=v$. Operator $J$ has precisely one antibound state at $\lambda_{0}$ of multiplicity 2 iff the discriminant given in (5.1) is zero. Moreover, the double antibound state different from $\lambda=v$ is given by either of the two following formula:

$$
\frac{\left(v+q_{2}\right) \pm \sqrt{\left(2 v-q_{2}\right)^{2}+3\left(a^{2}+1\right)}}{3} .
$$

The state $\lambda=v$ is always simple if $a \neq 0$.

Acknowledgement. The authors thank the referee for remarks. 


\section{References}

[1] J.E. Avron, A. Raveh, B. Zur. Adiabatic quantum transport in multiply connected systems. Rev. Modern Phys., 60 (1988), No. 4, 873-915.

[2] P. Exner. A duality between Schrödinger operators on graphs and certain Jacobi matrices. Ann. Inst. H. Poincaré Phys. Theor., 66 (1997), No. 4, 359-371.

[3] P. Harris. Carbon Nanotubes and Related Structures. Cambridge Univ. Press., Cambridge, 1999.

[4] A. Iantchenko, E. Korotyaev. Periodic Jacobi operators with finitely supported perturbations on the half-line. Preprint, 2009.

[5] S. Iijima. Helical microtubules of graphitic carbon. Nature, 354 (1991), 56-58.

[6] E. Korotyaev. Effective masses for zigzag nanotubes in magnetic fields. Lett. Math. Phys., 83 (2008), No 1, 83-95.

[7] E. Korotyaev. Resonances for Schrödinger operator with periodic plus compactly supported potentials on the half-line. Preprint, 2008.

[8] E. Korotyaev, A. Kutsenko. Zigzag nanoribbons in external electric Fields. To appear in Asympt. Anal.

[9] E. Korotyaev, A. Kutsenko. Zigzag and armchair nanotubes in external fields. To appear in Diff. Equations: Systems, Applications and Analysis. Nova Science Publishers, Inc.

[10] E. Korotyaev, I. Lobanov. Schrödinger operators on zigzag periodic graphs. Ann. Henri Poincaré, 8 (2007), 1151-1176.

[11] E. Korotyaev, I. Lobanov. Zigzag periodic nanotube in magnetic field. Preprint, 2006.

[12] P. Kuchment, O. Post. On the spectra of carbon nano-structures. Commun. Math. Phys., 275 (2007), 805-826.

[13] P. van Moerbeke. The spectrum of Jacobi matrices. Invent. Math., 37 (1976), No. 1, 45-81.

[14] D.S. Novikov. Electron properties of carbon nanotubes in a periodic potential. Physical Rev., B 72 (2005), 235428-1-22.

[15] L. Pauling. The diamagnetic anisotropy of aromatic molecules. J. of Chem. Phys., 4 (1936), 673-677.

[16] K. Pankrashkin. Spectra of Schrödinger operators on equilateral quantum graphs. Lett. Math. Phys., 77 (2006), 139-154. 
[17] V. Rabinovich, S. Roch. Essential spectra of difference operators on Zn-periodic graphs. J. Phys. A: Math. Theor., 40 (2007), 10109-10128.

[18] K. Ruedenberg, C.W. Scherr. Free-electron network model for conjugated systems. I. Theory. J. of Chem. Phys., 21 (1953), 1565-1581.

[19] R. Saito, G. Dresselhaus, M. Dresselhaus. Physical properties of carbon nanotubes. Imperial College Press, 1998.

[20] G. Teschl. Jacobi operators and completely integrable nonlinear lattices. Providence, RI: AMS, (2000) ( Math. Surveys Monographs, V. 72.)

[21] E.B. Vinberg. A Course in Algebra. Graduate studies in Mathematics, AMS, V. 56. 